\title{
La psicoterapia come scambio comunicativo. Prospettive di ricerca sul processo clinico
}

\author{
Sergio Salvatore, ${ }^{1}$ Alessandro Gennaro, ${ }^{1}$ Andrea Auletta, ${ }^{1}$ Rossano \\ Grassi, ${ }^{1}$ Stefano Manzo, ${ }^{1}$ Mariangela Nitti, ${ }^{1}$ Ahmed Al-Radaideh, ${ }^{1}$ Marco \\ Tonti, ${ }^{1}$ Nicoletta Aloia, ${ }^{1}$ Grazio Monteforte, ${ }^{1}$ Omar Gelo ${ }^{1}$
}

\section{Sommario}

Il lavoro illustra le 3 linee di lavoro nell'ambito della process research su cui si concentra l'interesse degli autori: a) l'analisi concettuale delle premesse teoriche e metodologiche che fondano la ricerca sullo scambio clinico; b) la definizione di un modello generale del processo clinico; c) lo sviluppo di strategie di analisi dello scambio clinico coerenti con tale modello generale. Ciascuna linea di sviluppo viene presentata e discussa in ragione dei suoi presupposti concettuali, di alcuni dei risultati rilevanti che ha prodotto, cosi come delle prospettive future a essa associata.

\section{Parole chiave}

Ricerca di processo, teoria dei sistemi dinamici, idiografico-nomotetico, abduzione, analisi testuale, Discursive Flow Analysis

${ }^{1}$ Università del Salento

Corrispondenza: Sergio Salvatore

E-mail: sergio.salvatore@unisalento.it 


\section{Introduzione}

La process research è in fase di transizione. Un numero crescente di ricercatori, consapevoli dei limiti concettuali e metodologici degli impianti di ricerca tradizionali, sono impegnati nello sviluppo di proposte innovative, tanto sul piano della teoria che dei modelli di analisi. Questi movimenti testimoniano la vitalità dell'ambito di ricerca; essi tuttavia non sembrano essere giunti a un punto di maturazione tale da permettere l'emergere di una nuova prospettiva, capace di ricostruire su basi rinnovate la ricerca clinica sul processo terapeutico.

Il nostro gruppo di ricerca si inserisce in questa fase di transizione, con tre linee di ricerca. In primo luogo, l'analisi concettuale delle premesse teoriche e metodologiche che fondano la ricerca sullo scambio clinico. In secondo luogo, la definizione di un modello generale del processo clinico. In terzo luogo, lo sviluppo di strategie di analisi dello scambio clinico coerenti con tale modello generale.

Questo lavoro si propone di passare in rassegna queste tre linee di ricerca, illustrandone i presupposti concettuali, i principali risultati e le prospettive verso le quali si indirizzano.

\section{Analisi concettuale della ricerca di processo}

La process research ha prodotto, e continua a produrre, una massa imponente di risultati empirici, frutto di una varietà di approcci (studi single case, analisi cliniche intensive, studi naturalistici, etc.) e strategie di analisi (applicazione di strumenti standardizzati, approcci ermeneutici, analisi del discorso, etc.). Un simile patrimonio di dati non sembra tuttavia offrire risposte soddisfacenti agli interrogativi generali che ne motivano la produzione, vale a dire: perché e come la psicoterapia funziona.

Diversi ricercatori - alcuni già diversi anni fa - hanno evidenziato una serie di criticità concettuali e metodologiche cui attribuire simile situazione di debolezza euristica (Greenberg, 1991; Laurenceau, Hayes, 
\& Feldman, 2007; Russel, 1994; Stiles \& Shapiro, 1994). Queste analisi hanno il merito di aver chiarito come lo sviluppo della process research non passi per il solo accumulo di dati e per la definizione di procedure di analisi sempre più sofisticate. È necessario anche un ripensamento dei presupposti paradigmatici taciti che fondano, regolano e al contempo vincolano la produzione empirica e l'interpretazione dei risultati. La ricerca di processo necessita, in altri termini, di uno sforzo di analisi concettuale che permetta ai ricercatori di ridefinire gli interrogativi che la guidano (Dazzi, Lingiardi, \& Colli, 2006).

In questa direzione, alcuni di noi hanno focalizzato la propria attenzione su alcune questioni di fondo - la definizione dell'oggetto, la teoria del significato di riferimento, il problema della generalizzazione usualmente assunte per default - la cui esplicitazione ed elaborazione concettuale è invece a nostro modo di vedere essenziale per uscire dalle secche dell'empirismo acefalo in cui molta della process research - e più in generale della psicologia empirica (cfr. Smedslund, 1987) — si ritrova (Salvatore, 2006a). Dedichiamo a ciascuna di queste questioni uno dei successivi sottoparagrafi.

Che cosa è il "processo terapeutico"? Lo statuto concettuale dell'oggetto di analisi

Due persone stanno giocando alla roulette su due diversi tavoli. La prima ha raggiunto il casinò per riprendersi da un congresso di ricerca in psicoterapia alquanto noioso. L'altra è un giocatore professionista, che fa delle vincite al tavolo verde la fonte del proprio reddito.

Come è facile constatare, i due giocatori sono portatori di scopi molti diversi e tale differenza si rifletterà nelle rispettive modalità di gioco (organizzazione delle puntate, livelli di attenzione, reazioni al risultato, etc.). Sarebbe tuttavia inverosimile concludere che anche il meccanismo di funzionamento del gioco vari, in ragione della differenza tra gli scopi dei due giocatori. 
In realtà l'esempio mette in evidenza una prospettiva ampiamente condivisa nell'ambito della process research. I ricercatori di questo campo, infatti, tendono in modo tanto implicito quanto tendenzialmente unanime, a considerare il processo terapeutico come un oggetto specifico, dotato di un proprio modo di operare, distinto da altre forme di relazione umana, da comprendere nei termini della definizione di un modello peculiare del suo funzionamento. A ben guardare, questa prospettiva sostiene che una determinata forma di relazione umana acquista una specifica modalità di funzionamento in ragione degli scopi - ad esempio la psicoterapia - che la motivano.

Alcuni di noi hanno approfondito in una serie di lavori le implicazioni epistemologiche e teoriche connesse a simile impostazione (Salvatore, 2006a; Salvatore \& Valsiner, 2006, 2009; Salvatore, Valsiner, Strouth, \& Clegg, 2009; Salvatore, Valsiner, Travers Simon, \& Gennaro, 2010a, 2010b), evidenziando i problemi che derivano - per la ricerca clinica, e più in generale per la psicologia - dall'adesione alla definizione di senso comune dei fenomeni assunti a oggetto di investigazione. A tali lavori rimandiamo per un approfondimento di questa linea di argomentazione, che in questa sede, per ovvi motivi di spazio, ci limitiamo a richiamare nei termini di un paradosso: se gli scopi socialmente definiti che motivano e orientano una determinata forma di relazione umana fossero in grado di configurarne il funzionamento, sarebbe allora necessario pensare a un'area di studi e ricerche distinta per ciascuna pratica socialmente definita. Dovremmo ad esempio avere teorie relative a: andare in pizzeria, visitare un museo, partecipare alle riunioni condominiali, implicarsi in una relazione sentimentale, giocare a golf, andare allo stadio, e così via. Certo, si potrebbe obiettare che non tutti questi processi sono rilevanti al punto da giustificare un settore di ricerca dedicato. Ma tale obiezione complicherebbe ancora di più la faccenda, in quanto, senza negare il carattere modello-specifico del fenomeno, implicherebbe, in aggiunta, che sia la gerarchia dei valori e 
degli interessi socialmente definiti a dettare l'agenda di ciò che va considerato meritevole di interesse scientifico.

Secondo la nostra tesi, la messa in discussione della specificità del processo psicoterapeutico non riduce, ma amplifica le possibilità euristiche della process research. Secondo questa prospettiva, il processo clinico si declina come manifestazione locale di un oggetto generale: lo scambio comunicativo (Salvatore, 2006a; Salvatore, in press). Questa concezione si basa su una distinzione tra la dinamica di un determinato oggetto e il processo che la invera (Salvatore \& Valsiner, 2010). La dinamica ha a che fare con il modo in cui funziona l'oggetto. In quanto tale, essa segue una modalità atemporale ed invariante - si ripete sempre uguale a se stessa; conseguentemente, si presta a essere modellizzata in termini di regole astoriche ed universali. D'altra parte, lo scambio comunicativo si realizza necessariamente entro specifici contesti socio-culturali, che qualificano gli scopi, dunque le condizioni e i vincoli di felicità dello scambio comunicativo. Tali condizioni e vincoli definiscono i termini entro, e attraverso i quali, la dinamica della comunicazione si invera. La stessa dinamica dà dunque luogo a processi diversi, in ragione dei parametri contingenti che definiscono le modalità del suo inveramento.

L'analogia con la fisica torna utile per illustrare il punto. Tale scienza si occupa di oggetti generalizzati, astratti dal loro contenuto empirico contingente - ad esempio: l'attrazione gravitazionale. Il modo del funzionamento (nei termini da noi sopra adottati: la dinamica) di tali oggetti è modellizzato nei termini di leggi universali invarianti — ad esempio, la teoria della relatività generale. Anche se la dinamica è invariante, essa dà luogo a processi tra loro molto diversi, in ragione delle condizioni di campo (nei termini da noi sopra adottati: in ragione dei parametri contingenti) in gioco. La traiettoria di un proiettile, lo slalom tra i paletti dello sciatore, il volo di un uccello, l'orbita di un pianeta e così via, sono esempi di fenomeni che riflettono la stessa 
fondamentale dinamica e al contempo si qualificano come processi diversi, in ragione dei parametri che intervengono nel loro inveramento. I diversi processi possono essere studiati localmente, vale a dire in ragione del loro contenuto empirico contingente. Ciò è quanto fanno discipline come la balistica, l'astronomia, l'idraulica, che si occupano di specifici campi fenomenici. Tuttavia, il funzionamento di tali campi fenomenici è sempre e comunque il riflesso della stessa dinamica generale. Il che equivale a dire che la traiettoria di un proiettile non è un oggetto che segue regole proprie e idiosincratiche rispetto alla traiettoria di uno sci. Ciò che rende tali processi diversi, lo ribadiamo, sono i parametri di campo. Conseguentemente, l'analisi della traiettoria del proiettile non può prescindere dalla comprensione della dinamica che qualifica il proiettile in quanto istanza dell'oggetto generalizzato "massa". Il che in altri termini significa che la modellizzazione della dinamica generale definisce il fondamento concettuale ed euristico per lo studio dei processi locali.

Quanto sopra detto porta a concludere che la psicoterapia è uno dei possibili processi che invera la comunicazione umana in ragione di parametri derivati in parte dal contesto culturale e istituzionale (il format professionale, il valore socialmente definito degli scopi, le forme organizzative) e in parte della teoria clinica (i parametri tecnici). Tali parametri rendono la psicoterapia una versione particolare dell'oggetto scambio comunicativo, differenziabile dalle infinite altre versioni dello stesso oggetto - corteggiamento, scrivere articoli scientifici, partecipare a una riunione, educare i figli, etc. La psicoterapia funziona in un certo modo non in quanto è un oggetto dotato di una propria modalità di funzionamento, ma perché riflette, in ragione di specifici parametri di campo, la dinamica fondamentale della comunicazione umana. Comprendere la psicoterapia, di conseguenza, richiede: a) la modellizzazione di tale dinamica; b) la comprensione dei parametri di 
campo che la inverano; c) la descrizione dell'organizzazione del processo che deriva da tale inveramento.

La distinzione dinamica/processo e la tesi della non autonomia della psicoterapia non è una questione da delegare alla discussione filosofica. Al contrario, è immediatamente rilevante per la process research in quanto da essa derivano implicazioni cogenti di ordine concettuale e metodologico. Ci limitiamo di seguito a richiamare, in estrema sintesi, due di queste implicazioni. In primo luogo, tale tesi implica che $\mathrm{i}$ meccanismi che rendono clinicamente rilevante lo scambio clinico siano gli stessi che sottendono le altre forme di comunicazione umana. Ciò equivale a dire che la risposta allinterrogativo del perché un determinato aspetto del processo terapeutico (ad esempio, la durata del trattamento, una caratteristica del paziente, una modalità di intervento del terapeuta, la qualità della relazione, etc.) incide nel modo in cui incide, vada ricercata nel modo con cui lo scambio comunicativo, in quanto tale, funziona.

Per inciso, quanto appena detto implica una rivisitazione della classica distinzione tra fattori aspecifici e specifici. In definitiva, gli aspetti del processo che incidono sul trattamento - siano essi prescritti o meno dalla tecnica - funzionano comunque in ragione di meccanismi fondamentali. Ciò significa reinterpretare l'opposizione specifico-non specifico nei termini della coppia specifico-generale.

In secondo luogo, dal punto di vista metodologico, la tesi proposta suggerisce la possibilità di una più estesa e sistematica adozione di metodi e strumenti di analisi elaborati in domini di ricerca diversi dalla clinica.

\section{Quale concezione del significato?}

Il processo terapeutico è una situazione di scambio comunicativo. Per chi come noi concepisce la relazione terapeutica in chiave dialogica (Gennaro \& Salvatore, 2010), lo scambio comunicativo è la sostanza 
stessa del processo clinico. Tuttavia, anche chi non condivide questa concezione non dovrebbe avere difficoltà a riconoscere nello scambio comunicativo il vettore del fattore clinico - vale a dire il veicolo di ciò che rende terapeutica la psicoterapia. In definitiva, il terapeuta opera nei termini di atti (linguistici, ma non solo) che possono avere una qualche incidenza sul paziente nella misura in cui quest'ultimo in qualche modo li raccoglie e interpreta nella loro dimensione di eventi comunicativi (Austin, 1962).

Quest'ordine di considerazioni ha spinto alcuni di noi ad approfondire la concezione del significato che si assume, generalmente in modo implicito, a fondamento del modo con cui si intende lo scambio comunicativo e dunque il processo terapeutico. Tale sforzo si muove su un piano generale e fondativo, dunque trasversale a una pluralità di domini di ricerca (per quanto più strettamente riferibile agli interessi di chi scrive: la ricerca clinica, ma anche la teoria generale della mente, l'analisi psicosociale del comportamento economico, dei setting formativi, organizzativi e socio-istituzionali; cfr. Forges Davanzati, Potì, \& Salvatore, 2008; Salvatore, in press; Salvatore, Forges Davanzati, Potì, \& Ruggeri 2009; Salvatore \& Freda, 2010; Salvatore, Freda, Ligorio, Iannaccone, Rubino, Scotto di Carlo, Bastianoni, \& Gentile, 2003; Salvatore, Tebaldi, \& Potì, 2006/2009; Salvatore \& Venuleo, 2008; Salvatore \& Zittoun, in press; Venuleo \& Salvatore, 2008).

Obiettivo centrale e qualificante di questa linea di ricerca è lo sviluppo e validazione empirica di una teoria dinamica del significato e della significazione (sensemaking). Tale modello si discosta dalla visione di senso comune, condivisa comunque anche da molta ricerca psicologica e psicologico clinica, che assume i significati come entità statiche, invarianti, proprietà fisse e discrete che si applicano agli oggetti rappresentati. Una pluralità di sviluppi del pensiero psicologico contemporaneo, in particolare i riscontri prodotti da un'ampia gamma di teorizzazioni che possiamo far rientrare entro la cornice concettuale 
del socio-costruttivismo (cfr. inter alia Bruner, 1990; Cole 1996; Gergen, 1999; Edwards \& Potter, 1992; Valsiner \& Rosa, 2007), hanno evidenziato la necessità di centrare l'analisi dei processi psicologici sull'attività interpretativa degli attori, processo entro e per mezzo del quale il significato viene co-costruito, piuttosto che meramente applicato. Il socio-costruttivismo ha messo in discussione la visione dei significati come entità fisse dell'universo simbolico, opponendo a essa l'idea secondo la quale essi non preesistono allo scambio sociale e comunicativo, ma sono costruiti e continuamente ridefiniti attraverso e in funzione di tale scambio. I significati sono un prodotto contingente della negoziazione intersoggettiva; tali negoziazioni, d'altra parte, piuttosto che rispondere esclusivamente a regole astratte, sono esse stesse atti sociali, orientati e organizzati da intenti pragmatici e retorici di regolazione dello scambio sociale.

In altra sede (Forges Davanzati et al., 2008; Salvatore, Forges Davanzati et al., 2009) alcuni di noi hanno discusso alcune caratteristiche del significato che il modello sopra accennato porta a evidenziare. Le richiamiamo brevemente di seguito, segnalando di volta in volta le implicazioni per la clinica.

Contestualità. Il sensemaking non è il prodotto di operazioni mentali chiuse e concluse entro la testa degli individui. Al contrario, esso è un processo intrinsecamente sociale, che si dispiega entro e attraverso 1o scambio comunicativo. Le strutture semantiche (i frame, gli schemi mentali, i copioni di azione, le matrici decisionali) che organizzano il funzionamento mentale non vanno intese in senso kantiano - cioè come forme a priori inscritte nella struttura della mente degli esseri umani; piuttosto, esse vanno concepite come prodotti storici, artefatti simbolici che la cultura di un determinato gruppo sociale configura e mette a disposizione dei propri membri (Cole, 1996). Dal punto di vista clinico, ciò significa che quanto accade entro il processo terapeutico va 
considerato sempre e comunque in ragione del contesto socio-simbolico in cui si inscrive, piuttosto che espressione immanente di una mente isolata e in se stessa autonoma.

Situatività. I significati non risiedono in una sorta di universo ubiquitario, dal quale condizionano i pensieri e i discorsi delle persone. Al contrario, sulla scorta della lezione di Wittgenstein (1958), siamo in condizione di riconoscere come essi si definiscano attraverso il modo con cui le persone usano i segni - dunque, in definitiva: del modo con cui agiscono (Harrè \& Gillet, 1994). Ciò vuol dire che i significati vanno considerati come circolarmente connessi alle circostanze della comunicazione e dell'agire. I significati, da un lato, permettono agli attori di comunicare e agire; dall'altro, sono sistematicamente e ricorsivamente ridefiniti da tale agire e comunicare. $\dot{E}$ in questo senso che parliamo di situatività dei significati ai discorsi: per evidenziare come i modelli simbolici non preesistono alla comunicazione ed all'azione, ma sono proprietà emergenti di tali processi (Salvatore, Tebaldi, \& Potì, 2006/2009; Salvatore \& Freda, 2010), precipitato delle forme situate di regolazione dello scambio sociale (Gergen, 1999; Grasso, Salvatore, \& Guido 2004; Salvatore, Ligorio, \& De Freanchis, 2005). Il riconoscimento del carattere situato del significato ha una conseguenza rilevante sul piano clinico e della process research. Le strutture di significato sovraordinate (frame) che regolano il pensiero si definiscono localmente, cioè all'interno ed attraverso le dinamiche micro-sociali in cui vengono utilizzate. Conseguentemente, per comprendere il senso di ciò che accade e viene comunicato entro il processo clinico non ci si può limitare a prendere in considerazione le singole unità della comunicazione, come se fossero entità in sè significative; va tenuto necessariamente in conto il qui ed ora della dinamica micro-sociale che sostanzia lo scambio clinico. E questo il principio metodologico dell'indessicalità della comunicazione (Salvatore, 
Gennaro, Grassi, Manzo, Melgiovanni, Mossi, Olive, \& Serio, 2007). Tale principio afferma che il significato di un atto - nel nostro caso, di un atto prodotto entro il processo clinico - può assumere una pluralità di significati, diversificati in ragione del contesto intersoggettivo ove si esercita; ad esempio, la stessa frase può risultare in un'offesa, una affermazione priva di senso, un apprezzamento - in ragione delle circostanze discorsive e relazionali entro cui è prodotta.

Pragmaticità. Il modo di pensare non è mai un'operazione neutrale; al contrario, è sempre e comunque un atto sociale, animato da una qualche forma (comunicativa, espressiva, argomentata che sia) di intenzionalità. In altri termini, il modo con cui gli attori danno significato all'esperienza è una delle leve fondamentali attraverso cui essi salvaguardano e promuovono reciprocamente le proprie prospettive, versioni del mondo, sistemi di interessi; in ultima istanza, il proprio ancoraggio identitario. Il che significa che le persone quando pensano e discutono non si limitano ad applicare schemi di significato in modo asettico, orientate da criteri di verità ciechi rispetto alle conseguenze. Al contrario, esse organizzano i pensieri, adottano strategie retoriche, assumono posizionamenti discorsivi ed intraprendono percorsi di costruzione di senso per accreditare/affermare un punto di vista, dunque per regolare lo scambio sociale in cui sono inscritti. In questo senso, pensare e parlare sono atti intrinsecamente sociali. Da ciò consegue una fondamentale implicazione euristica e metodologica: la necessità di considerare la dimensione pragmatica del significato. Aspetto per certi versi ovvio, ma non sempre tenuto in debito conto entro la process research, dove è ancora prevalente il focus esclusivo sulla componente semantica e/o sintattica del linguaggio (Manzo, 2010). 
Modalità di costruzione della conoscenza. Il problema della generalizzazione

Il processo terapeutico è per definizione un evento singolare, che vede implicate due (o più) persone per un periodo di tempo più o meno lungo. Lo studio scientifico, d'altra parte, richiede che le conoscenze relative ai singoli fatti travalichino i confini della singolarità in modo da rendersi generalizzabili.

Questa dialettica tra unicità e generalità è stata affrontata da alcuni di noi nei termini di una rilettura critica della classica opposizione nomotetico-idiografico (Salvatore, 2006b; Salvatore \& Valsiner, 2009; Salvatore, Valsiner, Strout, \& Clegg,, 2009; Salvatore, Valsiner, TraversSimon, \& Gennaro, 2010a, 2010b; si veda anche Molenaar \& Valsiner, 2009). Secondo l'originaria tesi di Windelband (1904/1998; cfr. anche Lamiell, 2003), i due termini sono in rapporto di complementarietà, piuttosto che di opposizione, come invece generalmente si ritiene. Data la loro natura dinamica (intesa come dipendenza temporale) e contestuale, gli oggetti psicologici sono singolari, nel senso che la relazione tra il loro modo di funzionare e le occorrenze fenomeniche nei termini delle quali tale modo si esprime, è mediata dalla contingenza delle condizioni di campo. Conseguentemente, la psicologia scientifica non può che essere idiografica, nel senso che non può che prendere in considerazione fenomeni unici e irreversibili (su questo punto, si veda anche Toomela, 2009, 2010). Allo stesso tempo, tuttavia, gli obiettivi di qualsiasi disciplina scientifica, dunque anche della psicologia, sono necessariamente nomotetici, volti cioè a costruire conoscenze generali, che trascendano l'ambito fenomenico specifico entro cui sono elaborate.

Il problema che dunque si pone alla psicologia, e quindi alla ricerca di processo, è quale modello logico di generalizzazione sia coerente con la natura idiografica del suo oggetto. In una serie di lavori recenti (Salvatore \& Valsiner, 2009, 2010) si è argomentato in favore della abduzione quale fondamentale forma della conoscenza psicologica. 
Ricerca in Psicoterapia / Research in Psychotherapy 2010; 2(13): 241-286 http://www.researchinpsychotherapy.net

Secondo la tesi proposta, l'unicità degli oggetti psicologici invalida la possibilità di fondare su base induttiva la generalizzazione, vale a dire in termini di accumulo di occorrenze empiriche provenienti da una pluralità di casi individuali (Peirce parla dell'induzione nei termini di acquisizione di una abitudine: se un evento occorre $n$ volte, allora se ne induce che si tratta di una regolarità che ci si abitua a considerare valida anche in futuro). Infatti, se i casi individuali sono per definizione incommensurabili, se ne deve inevitabilmente concludere l'impossibilità di assimilare tra loro le occorrenze relative a casi differenti. Questo stesso principio è stato concettualizzato in chiave psicometrica da Molenaar (2004; Molenaar \& Valsiner, 2009), nei termini del carattere non ergodico dei processi psicologici (su questo punto si rimanda a Salvatore, 2006b).

L'alternativa alla generalizzazione induttiva è l'abduzione (Di Nuovo, 2010). La generalizzazione fondata su tale logica parte, come l'induzione, dal dato, ma si orienta alla costruzione di un modello teorico locale, vale a dire un modello che interpreta (abbraccia in una totalità, secondo l'immagine di Peirce) le occorrenze fenomeniche del caso. Il modello teorico locale viene prodotto in ragione, ed entro i vincoli della teoria generale che guida l'investigazione abduttiva. È la relazione tra teoria locale e teoria generale a essere oggetto della generalizzazione. Ciò equivale a dire che la generalizzazione abduttiva concerne la costruzione di un modello locale che rifletta la teoria generale e sia allo stesso tempo sufficientemente astratto (cioè non espresso in termini dipendenti dal contenuto empirico contingente al singolo caso $^{1}$ ), per poter interpretare (abbracciare in una totalità) una pluralità di casi.

1. Si prenda come esempio il caso di un processo caratterizzato dalle occorrenze: $a, b, b, a, b, b, b, a, b, b$, $\mathrm{b}, \mathrm{b}, \mathrm{a}$. Il contenuto empirico di tale processo è unico per definizione, per cui non sarebbe possibile generalizzazione se fosse tale contenuto a essere assunto come oggetto di analisi. Al contrario, il pattern che caratterizza la relazione a-b può essere studiato oltre il (ma non indipendentemente dal) suo contenuto empirico - ad esempio come tendenza del secondo elemento della diade (b) ad aumentare la propria incidenza nel tempo. Ora, questo modello - che può anche essere formalizzato - è una mappatura astratta del caso, una sua rappresentazione priva di contenuto empirico. In questo modo diviene possibile creare una generalizzazione tra diversi casi attraverso un'operazione di astrazione — ad esempio si potrà 
Ricerca in Psicoterapia / Research in Psychotherapy 2010; 2(13): 241-286 http://www.researchinpsychotherapy.net

Approfondiamo brevemente quanto fin qui detto. Si consideri una serie $\mathrm{C}$ di casi $(1,2,3, \ldots, n)$. Si assuma $i_{x}$ come l'insieme di occorrenze espresse dal singolo caso. La generalizzazione induttiva definisce l'insieme (IC) degli insiemi di occorrenze, ix relativi ad ogni esemplare della serie $\mathrm{C}$, che sono (secondo gli standard e i presupposti dell'osservatore) descrittivamente tra loro simili. L'insieme $I_{C}$ è preso come indicativo della regola generale, valida per ogni caso di C.

Al contrario, nel caso della generalizzazione abduttiva, l'insieme $i_{1}$ (vale a dire: le occorrenze empiriche espresse dal caso 1 vengono modellizzate in quanto espressione di un fenomeno singolare e irripetibile, dunque non assimilabili alle occorrenze del caso 2. Conseguentemente, non si procede alla costituzione di $\mathrm{I}_{\mathrm{C}}$, ma si assume come base dati $i_{1}$. La modellizzazione delle occorrenze $i_{1}$ genera il modello locale $\mathrm{L}_{1}$. Per inciso, $\mathrm{L}_{1}$ è sviluppato a partire, in ragione ed entro $\mathrm{i}$ vincoli di una teoria generale $\left(\mathrm{T}_{\mathrm{C}}\right)$. Il modello $\mathrm{L}_{1}$ viene successivamente utilizzato per interpretare le occorrenze $i_{2}$, vale a dire le occorrenze espresse dal caso 2 . In tale processo interpretativo, $\mathrm{L}_{1}$ viene inevitabilmente sottoposto ad astrazione, al fine di contemplare la specificità locale del nuovo caso. Ciò lo trasforma nel modello $\mathrm{L}_{(1,2)}$ : un modello più generale di $\mathrm{L}_{1}$ che, senza perdere la valenza di interpretante della relazione tra $T_{C}$ e $i_{1}$, sarà allo stesso tempo in grado di interpretare anche $i_{2}$. Nella misura in cui $\mathrm{L}_{(1,2)}$ si mantiene compatibile con $\mathrm{T}_{\mathrm{C}}$, allora esso può essere considerato un'estensione generalizzata di $\mathrm{L}_{1}$. L'applicazione ricorsiva di tale procedura abduttiva alla successione di casi $\mathrm{C}(1,2 \ldots \mathrm{n})$ produrrà il modello $\mathrm{L}_{(1,2, . . \mathrm{n})}$, che costituirà il modello locale generalizzato, dotato del livello di astrazione necessario per interpretare la specificità della serie dei singoli casi. ${ }^{2}$

La differenza fondamentale tra induzione e abduzione, in sintesi, sta nel fatto che la prima punta a rilevare ciò che è comune tra i casi,

dire che il caso che evidenzia il pattern $\mathrm{m}, \mathrm{n}, \mathrm{n}, \mathrm{m}, \mathrm{n}, \mathrm{n}, \mathrm{n}, \mathrm{m}, \mathrm{n}, \mathrm{n}, \mathrm{n}, \mathrm{n}, \mathrm{m}$, pur avendo contenuto empirico differente, segue lo stesso modello di funzionamento del primo caso.

${ }^{2}$ Un esempio di modalità di ricerca che richiama questa procedura logica è dato dalla Task Analysis (cfr. Pascual-Leone, Greenberg \& Pascual-Leone, 2009). 
mentre la seconda ricerca il potenziamento della teoria attraverso l'accomodamento della stessa, alimentato dal confronto con la variabilità locale dei singoli casi.

Un esempio può aiutare a illustrare la differenza tra i due modelli di conoscenza sopra richiamati. Prendiamo in considerazione due ricercatori interessati a studiare la relazione tra l'andamento della sintomatologia e gli interventi del terapeuta. Poniamo ora che il primo ricercatore, Indut, segua la logica induttiva. Indut prende in esame il caso, da un lato verificando la presenza di interventi del terapeuta (poniamo, per semplicità, la frequenza degli interventi per seduta), dall'altro rilevando il livello di sintomatologia in determinati punti temporali (ad esempio, al termine di ogni seduta). Immaginiamo che Indut riscontri che il livello della sintomatologia sia minore nelle sedute dove si registra un numero più elevato di interventi del terapeuta. A questo punto, Indut passa a studiare una successione di ulteriori casi, trovando nella maggior parte delle circostanze (o in tutte, la differenza è qui irrilevante) risultati che considera tra loro simili e che dunque assimila nella seguente rappresentazione: "Nei casi analizzati ho osservato sistematicamente che quando aumenta la frequenza degli interventi del terapeuta diminuisce il livello di sintomatologia”. Avendo accumulato un numero consistente di osservazioni che ribadiscono tale associazione, Indut si sente legittimato (è indotto) a concludere che la relazione tra la frequenza degli interventi del terapeuta e la riduzione della sintomatologia sia una regola universale, valevole nella totalità dei casi. Indut ha operato così una generalizzazione induttiva. Per dirla nei termini sopra utilizzati, Indut ha estratto dai casi dell'insieme $\mathrm{C}$ l'insieme ridondante di occorrenze $i_{x}$ (andamento sintomatologia e frequenza interventi) presenti in tutti (o la maggior parte) dei casi C, e ha generalizzato $\mathrm{I}_{\mathrm{C}}$, dando a tale insieme il valore di rappresentazione di una legge valida per la generalità dei casi: $S=f(\operatorname{Int})$, che mappa la 
Sintomatologia (S) come funzione della frequenza degli Interventi (Int) del terapeuta.

Da quanto sopra osservato se ne ricava che la generalizzazione induttiva è una forma di conoscenza estensionale: una conoscenza che consiste nella possibilità di ampliare il numero di casi per i quali si considera valida una affermazione ricavata da - e originariamente riferibile a - un numero ristretto di casi.

Poniamo ora che Abdut, il secondo ricercatore, segua la logica abduttiva. Abdut assume come riferimento una teoria generale $\left(\mathrm{T}_{\mathrm{C}}\right)$, che precede (e dunque guida) l'osservazione empirica. Poniamo che Abdut sia una intersoggettivista e che dunque, assumendo la contingenza della mente del paziente alla relazione clinica, abbracci la teoria generale $S=f(R)$ : il livello della sintomatologia $S$ è una funzione della relazione $\mathrm{R}$ che si instaura tra paziente e terapeuta. Come si può osservare, il punto di partenza di Abdut è il punto di arrivo di Indut: la teoria generale (la $\mathrm{T}_{\mathrm{C}}$ da cui parte Adbut è tuttavia più astratta di quella a cui giunge Indut). Sulla scorta della $\mathrm{T}_{\mathrm{C}}$ di riferimento, Abdut avvia l'analisi, prendendo in esame il caso 1 . La $\mathrm{T}_{\mathrm{C}}$ la guida tanto nella selezione delle occorrenze pertinenti - la frequenza di interventi del terapeuta e il livello della sintomatologia - che nella modellizzazione delle relazioni tra esse. Abdut è così in condizione di formulare un modello interpretativo $\left(\mathrm{L}_{1}\right)$ del caso: $\mathrm{S}=\mathrm{f}(\mathrm{Int})$. Vale la pena evidenziare che tale modello, per quanto non differisca nel suo contenuto dal risultato dell'analisi di Indut, è prettamente locale, vale a dire è relativo e di validità circoscritta al caso 1: è una forma di conoscenza idiografica. Abdut procede nella sua analisi esaminando il caso 2, impegnandosi a interpretarlo nei termini del modello locale $\left(\mathrm{L}_{1}\right)$, così come precedentemente definito. Nel caso di 2, tuttavia, Abdut osserva un pattern che non si presta a essere assimilato a $L_{1}$ : in un certo numero, limitato, di sedute la maggiore frequenza degli interventi si associa a un incremento, piuttosto che a una riduzione del livello della 
sintomatologia. Tenendo conto dell'insieme delle occorrenze esaminate, questo pattern rappresenta un dato marginale, un'eccezione; ma è proprio su di esso che Abdut si concentra, "costringendosi" ad accomodare $\mathrm{L}_{1}$ in modo che tale modello sia in grado di "abbracciare" anche il pattern apparentemente divergente. Analizzando le occorrenze nella loro totalità, Abdut giunge così a formulare un nuovo modello locale $\mathrm{L}_{(1,2)}$, più generale del primo, in grado di interpretare 2 e allo stesso tempo di offrire una reinterpretazione del caso 1. Poniamo che tale modello sia $\mathrm{S}=\mathrm{F}\left(\mathrm{Int}_{\mathrm{rel}}\right)$ : il livello della sintomatologia dipende dalla proporzione di interventi del terapeuta rispetto agli atti linguistici prodotti dal paziente (Intrel). Come si può osservare, il modello $\mathrm{L}_{(1,2)}$ si colloca a un livello di astrazione maggiore di $L_{1}$, nel senso che: a) concerne un pattern maggiormente distante dal dato empirico contingente (ad esempio, lo stesso valore di Int può corrispondere a due

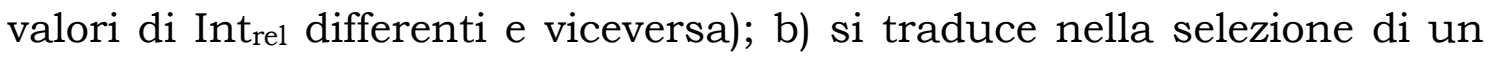
aspetto maggiormente circoscritto, frutto di un incremento del livello di selezione degli elementi ritenuti pertinenti (sul concetto di astrazione come pertinentizzazione si rimanda a Bühler (1934/1990); si veda anche Salvatore \& Valsiner, 2009). Abdut passa dunque al terzo caso, e poi ai successivi, di volta in volta accomodando il modello locale in ragione dei pattern divergenti. Ciò fino a quando il modello locale non risulti sufficientemente generalizzato da offrirsi come criterio interpretativo dei successivi casi senza necessità di ulteriori accomodamenti. Parallelamente, a mano a mano che il modello locale si generalizza e acquista progressivi livelli di astrazione, esso sottopone a "pressione" la teoria generale. Lo sviluppo del modello locale generalizzato, dunque, lavora come fattore di validazione o di ridefinizione della teoria generale, a seconda se quest'ultima sia in grado di "reggere" la pressione o si renda necessario il suo accomodamento. Possiamo così concludere che se la generalizzazione induttiva è una forma di conoscenza estensionale, la generalizzazione 
abduttiva ne è il contraltare intensionale: una forma di conoscenza consistente nel progressivo sviluppo (articolazione/astrazione) della teoria.

Anche in questo caso vale la pena evidenziare, sia pure in estrema sintesi, alcune implicazioni che rendono la discussione intorno all'abduzione di immediato interesse per la ricerca clinica. In primo luogo, la logica abduttiva restituisce il primato alla teoria sull'empiria; ciò senza tuttavia negare il valore dei dati, dunque della ricerca empirica. Al contrario, la ricerca empirica viene valorizzata come volano della costruzione teorica. Quanto sopra detto dovrebbe aver reso evidente, infatti, come la generalizzazione abduttiva sia guidata dalla teoria. Secondo tale modello, la conoscenza è un processo ricorsivo di sviluppo della teoria, precipitato dello sforzo sistematico di fondare interpretazioni locali di fenomeni (dunque di dati).

In secondo luogo, la logica abduttiva sollecita una strategia (e una cultura) di ricerca per certi versi opposta a quella canonica, basata sulla induzione. Lo sviluppo abduttivo della teoria richiede di mettere in tensione la valenza euristica della teoria. Conseguentemente, la scelta dei fenomeni da studiare si indirizza in ragione della ricerca dell'evento marginale, del dato divergente e sorprendente, quello che mette in discussione l'interpretazione acquisita, costringendo il ricercatore a rimodulare, rielaborare, astrarre la teoria. Insomma, i casi marginali che la logica induttiva considera rumore ostacolante la ricerca di regolarità, sono dalla logica abduttiva valorizzati come la fonte primaria di conoscenza.

Infine, la logica abduttiva rende evidente l'utilità in psicologia di pervenire a modalità di formalizzazione della conoscenza. Se la generalizzazione si esprime, secondo questa logica, nella progressiva astrazione del modello, allora è evidente che i linguaggi formalizzati si offrono come un utile strumento di sviluppo della teoria psicologica. 


\section{Per una teoria generale del processo}

In precedenti lavori alcuni di noi hanno evidenziato la necessità di distinguere due diversi obiettivi e focus di analisi: la ricerca nel processo versus la ricerca sul processo (Manzo, 2010; Salvatore, 2006b; Salvatore, Gelo, Gennaro, \& Manzo, 2009). Il primo tipo di ricerca si focalizza su specifiche dimensioni-costrutti ritenuti rilevanti dal punto di vista clinico (ad esempio: l'alleanza terapeutica, gli interventi del terapeuta, i meccanismi di difesa, il funzionamento metacognitivo). Il processo, secondo questa prospettiva, è il contenitore entro cui tali variabili si dispiegano. In definitiva, in quanto tale, il processo clinico non costituisce l'oggetto di analisi di questo tipo di ricerca, quanto il suo presupposto: lo scenario entro e grazie al quale la dimensionecostrutto target opera. Il secondo tipo di processo riflette un diverso, più generale, obiettivo: lo sviluppo di una teoria del processo clinico, inteso come un fenomeno in sé, da modellizzare nella sua globalità (Salvatore, Mossi, \& Gennaro, 2007). Simile teoria concerne interrogativi del tipo: in che cosa consiste lo scambio clinico? Come funziona? In che cosa consiste il cambiamento clinico? Quale dinamica lo sostanzia? Quali sono i vettori e i regolatori di tale dinamica?

In realtà, non sono molti i ricercatori che si sono proposti di definire una teoria generale del processo in grado di dare risposta a tale ordine di questioni (ad esempio, Mergenthaler, 1996; Bucci, 1997; Gonçalves, Matos, \& Santos, 2009). Ciò per certi versi è comprensibile: la ricerca sul processo è impresa complicata; data la pluralità delle forme e dei modelli psicoterapeutici, tale tipo di ricerca richiede l'elaborazione di modelli astratti generalizzati, tuttavia in grado di non disperdere la specificità del fatto clinico. D'altra parte, la definizione di una teoria generale del processo clinico è una priorità per la process research, che ha necessità di riferirsi a una cornice meta-teorica in grado di orientare e rendere reciprocamente commensurabili le analisi focalizzate sugli aspetti specifici dello scambio clinico. 
Sulla base di tali considerazioni, il nostro gruppo di ricerca si è in questi anni impegnato nella elaborazione e validazione di un modello generale del processo terapeutico (Gennaro, Al-Radaideh, Gelo, Manzo, Nitti \& Salvatore, 2010; Salvatore, Gelo, Gennaro, \& Manzo, 2009), basato su una concezione della mente di matrice dialogica e culturalista: il Two Stage Semiotic Model (TSSM).

\section{Il Two Stage Semiotic Model (TSSM)}

Il TSSM si basa su un postulato fondamentale e su tre assunti derivati da tale postulato.

Postulato fondamentale. La psicoterapia come dinamica di sensemaking. Lo scambio clinico è una dinamica intersoggettiva di costruzione di significato, finalizzata a modificare le modalità affettive e cognitive utilizzate dal paziente nell'interpretare le sue esperienze. I pazienti arrivano in psicoterapia dispiegando un sistema più o meno rigido di assunti dichiarativi e procedurali (concezioni di sé e degli altri, schemi affettivi, modalità metacognitive, strategie relazionali e di attaccamento, piani inconsci, etc) che fungono da significati sovraordinati, vale a dire da premesse di senso che regolano l'interpretazione dell'esperienza (Valsiner, 2007). Tali assunti rappresentano la fonte del problema che spinge in psicoterapia; allo stesso tempo essi sono la base e al contempo il vincolo all'attività di sensemaking. I sintomi, così come i conflitti intrapsichici e relazionali, sono concepibili come il sostrato e/o la conseguenza di tali significati sovraordinati. In ultima analisi, i significati sovraordinati sono il motivo, l'oggetto e l'obiettivo - così come il mediatore - della psicoterapia.

Assunto 1: Articolazione in due fasi. In un primo momento della psicoterapia il dialogo clinico (dunque l'incontro con un sistema di assunti altro, definito dal setting clinico) funge da limite al sistema di assunti del paziente. Se così non fosse il paziente non potrebbe che 
generalizzare le proprie modalità interpretative dell'esperienza alla relazione con il terapeuta, e così facendo riprodurre in modo assoluto all'interno del setting clinico quegli elementi critici per trattare i quali lo stesso setting clinico è stato disposto. Ciò comprometterebbe la capacità del dialogo clinico di introdurre aspetti innovativi nell'attività di sensemaking. Ad esempio, un paziente paranoico che considera l'altro pericoloso per default, avrebbe poche possibilità di usufruire della psicoterapia se, assimilando completamente il setting allo schema paranoico, fosse totalmente e assolutamente convinto che il terapeuta intenda danneggiarlo. In questa prospettiva, quindi, la prima fase dello scambio clinico si configura come un processo fondamentalmente decostruttivo, in cui il dialogo terapeutico funge da limite esterno all'attività regolativa dei significati sovraordinati (prevalentemente problematici) del paziente (Salvatore \& Valsiner, 2006).

L'indebolimento dei significati sovraordinati risultante dalla prima fase dello scambio clinico apre a un secondo momento, di tipo costruttivo, caratterizzato dall'elaborazione di nuovi assunti da parte del paziente. In questa seconda fase il dialogo paziente-terapeuta ha la possibilità di precipitare nella costruzione di nuovi significati sovraordinati, che possano fungere da regolatori innovativi nell'attività di sensemaking.

Ovviamente, le due fasi non sono totalmente separabili; tuttavia, a un livello macro-analitico, è possibile, in una psicoterapia a esito positivo, distinguere tra un primo momento caratterizzato da un processo di decostruzione e un secondo momento caratterizzato da un processo costruttivo, dove l'attività clinica funge da sostegno e impulso all'esplorazione, da parte del paziente, di nuovi significati.

Assunto 2: Non linearità del processo terapeutico. L'articolazione bifasica prospettata dal primo assunto del TSSM implica che lo scambio clinico svolge funzioni differenti in momenti differenti del processo clinico (fase decostruttiva e fase costruttiva). Conseguentemente, a 
differenza di quanto implicato nella visione tradizionale del processo terapeutico, la dinamica di sensemaking non segue un andamento lineare nel corso della psicoterapia: il sensemaking ha a che fare con il cambiamento di pattern di funzionamento, piuttosto che con un cambiamento cumulativo di elementi discreti tra loro indipendenti [sulla non linearità del processo clinico si veda, ad esempio, Russel (1994)].

Assunto 3: Quasi periodicità del sensemaking. Questo terzo assunto ha a che fare con il meccanismo micro-semiotico che istanzia la dinamica di sensemaking. In linea con la visione non lineare del processo clinico (Lauro-Grotto, Salvatore, Gennaro, \& Gelo, 2009), il TSSM assume un meccanismo quasi-periodico alla base dello scambio comunicativo. Tale meccanismo si qualifica per un andamento a strappi, simile al battito cardiaco, caratterizzato da momenti basici, rappresentativi del funzionamento del sistema di assunti del paziente, che vengono interrotti da momenti circoscritti di "irruzione" di variabilità semiotica, ovverossia di ricombinazione tra $\mathrm{i}$ diversi significati.

\section{Evidenze relative al TSSM}

Il nostro gruppo ha sviluppato un metodo di analisi del processo clinico coerente con il TSSM: il Discourse Flow Analysis (DFA), utilizzandolo nell'analisi di una successione di casi, secondo il modello della generalizzazione abduttiva descritto in precedenza. Rinviamo al prossimo paragrafo per la presentazione del metodo e l'illustrazione di alcuni risultati ottenuti tramite il suo uso. Qui ci limitiamo ad anticipare che $\mathrm{i}$ riscontri fin qui ottenuti si prestano ad essere interpretati nei termini del modello teorico, in questo modo offrendosi come elementi a sostegno della validità di costrutto del TSSM. In particolare:

a) Per ciascuno dei casi analizzati, l'andamento dell'incidenza dei significati sovraordinati segue una curva a U. Tale traiettoria è 
Ricerca in Psicoterapia / Research in Psychotherapy 2010; 2(13): 241-286 http://www.researchinpsychotherapy.net

interpretabile alla luce del TSMM, come successione di una fase decostruttiva, quando lo scambio clinico opera in modo da ridurre i significati sovraordinati di cui è portatore il paziente, seguita da una fase costruttiva, quando la psicoterapia si caratterizza per la capacità di sviluppare nuovi significati.

b) le analisi dei casi evidenziano come alle due fasi previste dal TSMM corrispondano pattern di funzionamento differenti. Ad esempio, svariate correlazioni tra le variabili rilevanti si modificano - sia in termini di entità che di direzione - nel passaggio da una fase all'altra.

c) Le analisi hanno messo in evidenza un andamento a strappi della dinamica di sensemaking. Questo tipo di andamento è interpretabile in ragione del terzo assunto del TSSM secondo il quale il sensemaking procede secondo un meccanismo caratterizzato dalla irruzione di momenti circoscritti di innovazione semiotica.

\section{Questioni di metodo}

Tra i ricercatori sta progressivamente diffondendosi la consapevolezza circa la necessità di tenere maggiormente in conto la complessità del processo clinico (Dazzi, 2006; Grasso, 2010). ${ }^{3}$ Tale consapevolezza sta

\footnotetext{
${ }^{3}$ In realtà il tema non è per nulla nuovo. Circa un quarto di secolo fa, Stiles e Shapiro (1994, pag. 37) proposero un critica radicale del paradigma di ricerca tradizionale che assimilava la ricerca in psicoterapia alla ricerca in campo farmacologico. Gli autori chiamarono tale assimilazione "drug metaphor", descrivendola nei termini seguenti. "[...] Un paradigma investigativo [...] [che] guarda alla psicoterapia come un composto di principi attivi forniti dal terapeuta al paziente [...] Tali supposti ingredienti attivi sono componenti di un processo - tecniche terapeutiche come l'interpretazione, la confrontazione , la riflessione, la self disclosure, la focalizzazione sulle emozioni, lo sforzo per dare supporto, o (più astrattamente) l'empatia, il calore o la genuinità. Se un componente è un ingrediente attivo allora una sua forte somministrazione è ritenuta un portare verso un esito positivo, in caso contrario l'ingrediente viene ritenuto inerte [...]" (ndr. traduzione nostra).

La drug metaphor è un esempio classico di processo lineare, molecolare e additivo. In linea con tale prospettiva: a) processo ed esito sono distinguibili, giacchè il primo causa il secondo; b) gli ingredienti del processo sono elementi conosciuti, sostanziali, isolabili, alla stregua di elementi discreti che vengono via via implementati in linea con procedure tecniche indipendenti e che hanno sempre lo stesso effetto sul paziente, nel corso del processo (Stiles \& Shapiro, 1994).

Questi assunti rappresentano un modello chiaramente ipersemplificato di psicoterapia, che scotomizza la natura contestuale, olistica, contingente e non lineare del setting clinico. Lo scambio clinico è caratterizzato da un numero elevato di fattori, molto superiore al numero di aspetti che la ricerca in psicoterapia è in grado di isolare (cfr. Contestualità, cfr. Bickhard, 2009). Inoltre, ciò che è rilevante non sono gli elementi in se stessi, ma la loro interazione, ovvero il modo in cui lavorano come parte di un
} 
Ricerca in Psicoterapia / Research in Psychotherapy 2010; 2(13): 241-286 http://www.researchinpsychotherapy.net

facendo emergere una domanda di strategie e metodi di analisi innovativi, in grado di sostituire gli approcci tradizionalmente adottati, implicanti una visione statica, molecolare e lineare dello scambio clinico. Il nostro gruppo di ricerca si propone di contribuire a questa prospettiva di innovazione metodologica. Tre sono in particolare le linee di ricerca, tra loro inevitabilmente intrecciate, che stiamo in questa direzione percorrendo.

In primo luogo, in una serie di lavori di rassegna (Gelo \& Salvatore, submitted;; Lauro-Grotto, Salvatore, Gennaro, \& Gelo, 2009; Salvatore, Lauro-Grotto, Gennaro, \& Gelo, 2008; Salvatore, Lauro-Grotto, Gennaro, \& Gelo, 2009; Salvatore \& Tschacher, submitted) e di analisi empiriche (Gennaro, Al-Radaideh, Gelo, Manzo, Nitti, \& Salvatore, 2010; Salvatore, Gennaro, Auletta, Grassi, \& Rocco, submitted; Auletta, Salvatore, Metrangolo, Monteforte, Pace, \& Puglisi, submitted), abbiamo proposto argomenti, esempi e dati a favore della Teoria dei Sistemi Dinamici come fonte di metodologie maggiormente coerenti con la natura di campo dello scambio clinico. In secondo luogo, stiamo lavorando allo sviluppo di sistemi automatici di analisi dei trascritti di seduta (Nitti, Ciavolino, Salvatore, \& Gennaro, 2010; Salvatore, Gennaro, Auletta, et al., submitted; Salvatore, Gennaro, Auletta, Tonti, et al., submitted). In terzo luogo, la concezione dinamica e contestuale del processo clinico delineata nelle pagine precedenti si è tradotta in un metodo di analisi - il DFA (Discourse Flow Analysis) - volto a permetterne la validazione.

tutto (Olismo, cfr. Valsiner, 2007; Salvatore \& Valsiner, in press). Di conseguenza nessun elemento può essere pensato come portatore di una valenza clinica invariante. Piuttosto, il suo impatto sul processo è mediato dal campo, inteso come l'insieme delle co-occorrenze di elementi (Non linearità; cfr. Barkham, Stiles, \& Shapiro, 1993). Inoltre l'idea di ingredienti tecnici implementati dal processo, ma indipendentemente dal processo, contrasta con l'ovvia osservazione clinica che il paziente non è soltanto il bersaglio ricettivo dell'azione del terapeuta ma anche un agente che a sua volta stimola l'azione del terapeuta (Contingenza, cfr. Goncalves, Ribeiro, Matos, Santos, \& Mendes, 2010). Infine come il dibattito sull'alleanza terapeutica evidenzia (Colli \& Lingiardi, 2009), l'unidirezionalità del legame tra processo ed esito non è più sostenibile: processo ed esito si associano in modo circolare - il primo causa ed è causato dal secondo (Circolarità; Greenberg \& Pinsof, 1986). 
Proprietà dello scambio clinico e nuove strategie di analisi

Il punto di partenza della nostra argomentazione è il riconoscimento della valenza di campo del significato (Salvatore, in press). Come già osservato, diversi approcci nel campo della psicologia, della semiotica, della linguistica, della filosofia hanno evidenziato come il significato sia un processo dinamico e situato che si snoda attraverso l'uso delle parole (e altri segni), piuttosto che una qualità invariante inerente $\mathrm{i}$ segni (inter alia, Andersen, 2001). Le parole acquistano significato in funzione delle specifiche circostanze socio-discorsive in cui vengono prodotte. Il riconoscimento del carattere di campo del significato ci ha portato a evidenziare due proprietà fondamentali dello scambio clinico - la sistematicità e la dinamicità - e a derivare da esse altrettante basilari indicazioni metodologiche: studiare le configurazioni; analizzare le sequenze.

Sistematicità. Il significato dei segni dipende dal modo in cui vengono utilizzati, da come si combinano con altri segni, allinterno delle circostanze discorsive (Greenberg \& Pinsof, 1986; Harré \& Gillett, 1994; Fornari, 1979). In questa prospettiva ciò che diviene rilevante non è tanto l'occorrenza dei segni, quanto la loro relazione. Freud (1900/1953) ha già sottolineato questo aspetto mettendo in guardia dal fornire interpretazioni semplicistiche dei simboli del sogno in termini di corrispondenza uno a uno tra simbolo e significato (ad esempio, sigaro $=$ pene). Similmente, il sensemaking è una attività di sistema in cui l'intera rete di relazioni tra elementi è diversa dalla loro composizione additiva: i significati sono come elementi chimici che producono entità diverse in ragione di piccole modifiche nella loro combinazione (Grassi, 2008).

Dinamicità. Sottolineare la valenza sistemica del sensemaking implica sottolinearne anche la sua natura intrinsecamente dinamica, ovvero la 
sua dipendenza temporale. Il linguaggio è per sua natura sequenziale; le relazioni tra i segni linguistici sono necessariamente relazioni temporali. Il tempo non è solamente il contenitore all'interno del quale il significato si dispiega; esso esercita un ruolo costitutivo nella costruzione del significato (Lauro-Grotto, Salvatore, Gennaro, \& Gelo, 2009; Nitti, Ciavolino, Salvatore, \& Gennaro, 2010; Salvatore, Lauro-Grotto, Gennaro, \& Gelo, 2009). Il sensemaking non ha a che fare solo con ciò che è detto e con come è detto; ma anche con quando ciò che è detto è detto, ovverossia prima o dopo che cosa. Si prendano ad esempio le seguenti affermazioni, che potrebbero caratterizzare la produzione narrativa di due ipotetici pazienti:

\section{Paziente 1}

"Quando perdo al gioco mi arrabbio molto e desidero essere aiutato da uno psicoterapeuta"

Paziente 2

"Quando desidero essere aiutato da uno psicoterapeuta mi arrabbio molto e perdo al gioco"

Le due frasi sono composte dalle stesse parole e sul piano del contenuto sono identiche; eppure il loro significato è notevolmente diverso, in ragione dell'ordine con cui le parole compaiono: mentre la prima frase riferisce del bisogno di supporto terapeutico associato all'esperienza di gioco, la seconda connota il gioco come dimensione di acting out, reattiva al riconoscimento del desiderio di aiuto.

Studiare le configurazioni. La natura sistemica del sensemaking porta a spostare il focus delle analisi dalle occorrenze di categorie discrete di significato alla loro combinazione in termini di pattern (von Eye, Mum, \& Mair, 2009; Greenberg, 1994; Matos, Santos, Gonçalves, \& Martins, 
2009; Salvatore, Lauro-Grotto, Gennaro, \& Gelo, 2009; Salvatore, Tebaldi, \& Potì, 2006/2009). Prendiamo ad esempio la presenza di tre categorie semantiche: $a, b$, e $c$, che occorrono rispettivamente 3,5 , e 2 volte. Se il nostro studio si limitasse all'analisi della loro distribuzione concluderemmo che $a$ e $b$ sono le più frequenti. Ciò tuttavia non necessariamente implica che queste categorie siano le più rilevanti. L'analisi del loro ruolo, infatti, richiede una mappatura di come le categorie si combinano l'una con l'altra. Ed è facile osservare come la stessa distribuzione complessiva possa corrispondere a una pluralità di scenari di combinazione (ad esempio, scenario 1: $a-a-b-b, c-c-a, b-b-b$; scenario 2: $a-a-a-b,-b-b-b, b-c-c)$, dunque a una pluralità di significati globali.

Analizzare le sequenze (di configurazioni). La dipendenza temporale del sensemaking porta a focalizzare l'analisi sui pattern diacronici, oltre che sincronici, di combinazione dei contenuti. Ciò significa porre attenzione alle transizioni tra i significati: quale contenuto segue o precede quale, con quale probabilità e in ragione di quali condizioni elicitanti. La transizione assume la funzione metodologica di marcatore della dinamica di sensemaking.

Diversi lavori del nostro gruppo di ricerca hanno adottato modalità di analisi ispirate ai due criteri metodologici appena richiamati. Ad esempio, Nitti e colleghi (2010) hanno utilizzato una procedura integrante l'analisi markoviana delle sequenze (cfr. anche Salvatore, Gennaro, Grassi, Manzo, Melgiovanni, Mossi, Olive, \& Serio, 2007; Salvatore, Lauro-Grotto, Gennaro, \& Gelo, 2009) e l'implementazione di una rete neurale; cosi facendo sono stati in grado di distinguere sedute clinicamente positive versus sedute non positive - così definite sulla base di un criterio clinico indipendente. Salvatore, Tebaldi e Potì $(2006 / 2009)$ hanno adottato un metodo di analisi basato sullo studio 
Ricerca in Psicoterapia / Research in Psychotherapy 2010; 2(13): 241-286 http://www.researchinpsychotherapy.net

della dimensionalità dello spazio delle fasi ${ }^{4}$ utilizzato per rappresentare l'andamento nel tempo della variabilità lessicale caratterizzante 1o scambio comunicativo durante una psicoterapia. In particolare, gli autori hanno riscontrato come la dimensionalità dello spazio si riduca drasticamente nel periodo immediatamente successivo all'inizio della psicoterapia, per poi conservare un andamento stabile fino al termine della terapia. Tale risultato è stato interpretato come indicativo dell'emergenza e successivo mantenimento di una cornice di senso condivisa entro lo scambio clinico, espressione di un accordo discorsivo tra terapeuta e paziente, che si riflette nella riduzione della libertà di associazione tra le parole (per un'analisi simile, basata sul riferimento teorico-metodologico alla sinergetica, si veda Gelo, Ramseyer, Mergenthaler, \& Tschacher, 2008).

Santos, Gonçalves, Matos e Salvatore (2009) hanno studiato la dinamica del cambiamento nelle narrazioni di una paziente, attraverso una procedura di analisi multidimensionale (Analisi delle Corrispondenze e Analisi dei Cluster) volta a estrapolare configurazioni narrative indicative di momenti di cambiamento nelle modalità discorsive del paziente (gli Innovative Moments secondo la terminologia del metodo utilizzato, cfr. Gonçalves, Ribeiro, Matos, Santos, \& Mendes, 2010). In questo modo l'analisi è andata oltre la mera rilevazione dell'incidenza delle singole categorie narrative, per concentrarsi sulla identificazione di combinazioni di occorrenze e sulla loro evoluzione lungo l'arco temporale del processo terapeutico analizzato.

Recentemente, Gelo \& Salvatore (submitted) e Salvatore \& Tschacher (submitted) hanno presentato una serie di strategie di analisi ispirate alla Teoria dei Sistemi Dinamici [analisi di Montecarlo, differenza mobile

\footnotetext{
${ }^{4}$ Lo spazio delle fasi è lo spazio ciascun punto del quale rappresenta uno e un solo stato del sistema descritto. Ad esempio, un punto di uno spazio delle fasi a due dimensioni, descriverà uno stato del sistema nei termini delle coordinate di tale punto. In generale, lo spazio delle fasi ha una dimensionalità corrispondente ai gradi di libertà del sistema che descrive. Ad esempio, se un corpo si muove esclusivamente su un piano, serviranno due dimensioni per descrivere la sua traiettoria; se invece si muove in uno spazio tridimensionale, serviranno tre dimensioni. La dimensionalità dello spazio delle fasi è dunque un indicatore della variabilità del comportamento dei sistema.
} 
(Salvatore, Serio, \& Manzo, 2006); modellizzazione delle traiettorie; studio delle probabilità di transizione; analisi univariata dei trend (Molenaar \& Valsiner, 2009)], argomentandole sul piano teorico-clinico ed illustrandole con esempi tratti da una varietà di ricerche.

Infine, Gelo e Salvatore (submitted), hanno discusso dati clinici e di ricerca che evidenziano il carattere dinamico, disomogeneo, non lineare, discontinuo e multi-direzionale del cambiamento terapeutico. Il riconoscimento di tali caratteristiche comporta la necessità di ripensare criticamente molti degli assunti che sono attualmente alla base della ricerca in psicoterapia, così come di sviluppare strategie di analisi focalizzate sullo studio intensivo dei casi.

\section{L'analisi dei trascritti}

Una delle fondamentali fonti della ricerca di processo è data dalle trascrizioni delle sedute. Lo sviluppo della process research passa dunque inevitabilmente per il potenziamento della validità e efficienza dei modelli e delle procedure di analisi testuale. In ragione di questa prospettiva, stiamo lavorando alla validazione di un metodo automatizzato di analisi del contenuto dei trascritti di seduta (Nitti, Ciavolino, Salvatore, \& Gennaro, 2010; Salvatore, Gennaro, Auletta, Tonti, \& Nitti, submitted)

A oggi i metodi automatizzati nella ricerca clinica sono pochi e limitati all'analisi della dimensione lessicale. Si tratta di metodi che implicano una concezione a-contestuale del significato, in ragione della quale ogni segno (una parola, una frase) viene associato a un set prefissato di parametri di valore, implementato tramite algoritmi automatizzati (ad esempio, si veda il TCM, Merghentaler, 1996). La contestualità del significato rende evidentemente poco praticabile l'estensione di tale strategia metodologica al piano semantico, la cui analisi, almeno nel campo clinico, è rimasta così affidata al lavoro interpretativo del ricercatore. 
Il ricorso al giudizio umano solleva tuttavia problemi organizzativi e metrici non indifferenti. Da un lato, nonostante l'assoggettamento del giudizio umano a regole di codifica estensibili e sistematiche, l'inferenza dei rater rimane comunque soggetta a una irriducibile valenza soggettiva. In conseguenza di ciò, i metodi per l'analisi semantica soffrono di bassi livelli di attendibilità, vincolo che riduce non marginalmente la loro capacità di rivelare relazioni significative. Non meno importante, l'analisi di contenuto è solitamente molto laboriosa: richiede tempo e risorse umane. E ciò rappresenta un ulteriore ostacolo all'applicazione dei metodi semantici allo studio del processo clinico.

Diversi criteri sono stati proposti per rispondere ai problemi che pone l'uso di tali metodi. Molti sforzi sono stati fatti per definire regole di codifica chiare e specifiche, che vincolino i giudici all'uso di procedure di validazione consensuale (Lutz \& Hill, 2009; Lambert, 2004); tuttavia, dato il livello di inferenza implicato in tali metodi, queste soluzioni non possono essere pienamente risolutive; esse, inoltre, rendono l'uso di metodi di analisi semantica ancora più dispendiosi.

\section{La Automated Co-occurrence Analysis for Semantic Mapping (ACASM)}

Sulla base di quest'ordine di considerazioni abbiamo deciso di avviare un programma di lavoro finalizzato a sviluppare una procedura automatizzata di analisi semantica (ACASM), in grado di ridurre il ruolo giocato dallinferenza umana; ma allo stesso tempo in grado di prendere in considerazione la dimensione contestuale del significato, dunque la sua indessicalità. Il nostro gruppo di ricerca è solo all'inizio di questo programma. Di seguito, richiamiamo brevemente la logica alla base del metodo sviluppato e i primi incoraggianti risultati di validazione ottenuti (Salvatore, Gennaro, Auletta, Tonti, \& Nitti, submitted)

L'Automated Co-occurrence Analysis for Semantic Mapping (ACASM) è un adattamento al campo della ricerca clinica di un modello di analisi testuale elaborato nel campo della statistica lessicale e già utilizzato in 
altri ambiti della ricerca psico-sociale (Carli \& Paniccia, 2002; Lancia, 2002). Il metodo si focalizza sulle co-occorrenze di parole, ovvero sul modo con cui le parole si combinano tra loro nelle unità di analisi in cui il testo viene scomposto (solitamente frasi o gruppi di frasi). La cooccorrenza di parole viene utilizzata quale espressione di un criterio di somiglianza per la clusterizzazione delle unità di analisi. In altre parole, le unità di analisi vengono ricomposte in cluster in base alle cooccorrenze di parole: le unità di analisi che tendono a contenere le stesse co-occorrenze di parole vengono considerate simili e quindi raggruppate.

L'idea alla base del metodo assume che un insieme di co-occorrenze determina uno specifico nucleo tematico; quindi, le unità che hanno un certo insieme di parole co-occorrenti condividono lo stesso nucleo tematico. In questo modo la procedura di analisi è in grado di fornire un livello di rappresentazione semantica del testo che codifica ogni unità di analisi nei termini di un determinato nucleo tematico.

L’ACASM si basa su algoritmi invarianti operazionalizzati da specifici software (Alceste, T-LAB). In particolare noi utilizziamo la procedura implementata da T-LAB (Lancia, 2002), nella versione T-LAB_PRO_XL2. Gli algoritmi implementano le diverse fasi del metodo nel seguente modo:

1) Segmentazione del testo in unità di contesto (sostanzialmente equivalenti alle frasi).

2) Costruzione del vocabolario delle unità lessicali presenti nel testo.

3) Rappresentazione digitale del testo, nei termini della matrice avente in riga le unità di contesto, in colonna le unità lessicali, nella cella ij-esima il valore $0 / 1$ indicativo della presenza/assenza della unità lessicale della colonna j-esima nella unità di contesto della riga i-esima. 
4) Analisi multidimensionale volta alla definizione di cluster di cooccorrenze di unità lessicali (e delle unità di contesto corrispondenti a tali co-occorenze).

5) Il lavoro interpretativo del ricercatore si focalizza su tali cluster e si dedica alla individuazione del nucleo tematico di cui ciascun cluster è, secondo la logica del metodo, marcatore.

E opportuno notare come la ACASM sia un metodo bottom up di analisi del contenuto: esso infatti non parte da un repertorio prestabilito di contenuti tematici in base ai quali vengono classificate le unità di testo; piuttosto, il repertorio di contenuti che funge da sistema di codifica è prodotto dal'analisi stessa, come risultato dell'identificazione di insiemi di parole co-occorrenti presenti nel testo. Da un punto di vista concettuale, il riferimento alle parole co-occorrenti all'interno della stessa unità di analisi può essere considerato un modo per prendere in considerazione la dimensione contestuale del significato, nella sua componente intratestuale.

La validazione di ACASM ha adottato un impianto di analisi ispirato al criterio di Turing. Si è assunto che sarebbe stato possibile considerare valido il metodo automatizzato di codifica del contenuto, nella misura in cui i risultati della sua applicazione non fossero risultati distinguibili da quelli prodotti da codificatori umani. Il confronto tra ACASM e giudici esperti è avvenuto sui trascritti di una psicoterapia (Gennaro \& Salvatore, in press; Salvatore, Gennaro, Auletta, Tonti, \& Nitti, submitted). Conformemente alle aspettative, $i$ risultati hanno evidenziato come la ACASM produca una mappatura del contenuto tematico dei trascritti delle sedute di psicoterapia non differenziabile da quella prodotta da giudici indipendenti e ciechi.

Il DFA: la mappatura della dinamica dello scambio clinico

Da alcuni anni (Gennaro, 2008; Gennaro, Melgiovanni, \& Serio, 2007; Gennaro, Al-Radaideh, Gelo, Manzo, Nitti, \& Salvatore, 2010; 
Nitti, Ciavolino, Salvatore, \& Gennaro, 2010; Salvatore, Grasso, \& Tancredi, 2004; Salvatore, Gennaro, Manzo, Melgiovanni, \& Serio, 2007; Gennaro et al., 2010; Salvatore, Gennaro, Lis, Di Riso, Laghezza, \& Sbabo, 2006) siamo impegnati nello sviluppo di un metodo di analisi del processo terapeutico in termini di dinamica discorsiva (Discursive Flow Analysis, originariamente denominato RIFLUD: Rivelatore dei Flussi Discorsivi). Tale metodo riflette i principi teorici e metodologici (ricerca sul processo, approccio contestuale e dinamico, generalizzazione abduttiva) che abbiamo illustrato nella prima parte di questo scritto. Potremmo dire che, coerentemente con la logica di generalizzazione abduttiva, l'elaborazione di tali principi ha alimentato ed al contempo è stata alimentata dal progressivo sviluppo del metodo e dal suo uso nell'analisi di casi di psicoterapia.

Il DFA si inscrive nella cornice teorica del TSSM, che qualifica la psicoterapia quale dinamica tesa alla produzione di innovazione semiotica. Il DFA adotta una procedura di analisi semi-automatizzata che combina tecniche di analisi del testo e di statistica multidimensionale. Sulla base di tale procedura, il DFA identifica i principali significati attivi nel discorso tra paziente e terapeuta e mappa la struttura e la dinamica della loro combinazione. Più in particolare, il DFA descrive la dinamica delle interconnessioni tra i significati attivi nello scambio clinico in termini di Rete Discorsiva. La Rete Discorsiva può essere analizzata sia quantitativamente che qualitativamente. L'analisi quantitativa si basa su una serie di indici (Attività, Connettività, Nodi sovraordinati) che permettono di stimare la forza dinamica della rete (la sua capacità di generare significati innovativi), così come la sua struttura (il livello e la natura delle connessioni tra i significati attivi nel flusso discorsivo). L'analisi qualitativa concerne l'interpretazione clinica del contenuto dei significati corrispondenti ai nodi della rete (Gennaro, 2008). 
Il DFA è stato applicato all'analisi di casi di psicoterapia di diversa lunghezza e diverso orientamento, con lo scopo di studiarne la validità, sia di costrutto, sia relativa a un criterio. Per quanto riguarda la validità di costrutto, gli indici dinamici e strutturali del metodo si sono mostrati capaci di offrire una rappresentazione dello scambio comunicativo tra paziente e terapeuta sensata dal punto di vista clinico e coerente con il TSSM, che ne costituisce la cornice concettuale. Per quanto riguarda la validità di criterio, i risultati principali sono così sintetizzabili:

a) Salvatore, Gennaro, Grassi e colleghi (2007) hanno applicato il DFA a una psicoterapia a esito positivo (caso di Katja; Dimaggio \& Semerari, 2001; Dimaggio, 2007) di 124 sedute. Gli indici del DFA risultano correlare con gli indici IVAT (Indice di Valutazione dell'Alleanza Terapeutica; cfr. Colli \& Lingiardi, 2002) e con alcune delle scale della DMRS (Defense Mechanism Rating Scale; Perry, 1990) - per l'applicazione di ambedue gli strumenti al caso Katja si veda Lingiardi, Colli, \& Gazzillo, 2007). Tali correlazioni sono state interpretate come un elemento a riscontro della capacità del DFA di cogliere specifici andamenti clinici (alleanza terapeutica e modifica dei pattern difensivi).

b) Un successivo lavoro, basato sull'analisi di un caso di psicoterapia breve (caso di Lisa, 15 sedute; Gennaro, Melgiovanni, \& Serio, 2007) ha verificato la capacità del DFA di discriminare tra le sedute ritenute clinicamente positive e non positive - definite tali sulla base di un criterio esterno indipendente: la presenza o l'assenza di un ciclo terapeutico individuato attraverso il TCM (Merghentaler, 1996). L'interpretazione degli indici del DFA in termini di pattern ha permesso di discriminare tra le due categorie di sedute con una percentuale di successo del 100\%.

c) Gennaro, Gonçalves, Mendes, Ribeiro e Salvatore (in press) in uno studio di convergenza tra il DFA e l'Innovative Moment Coding System (IMCS; Goncalves, Ribeiro, Matos, Santos, \& Mendes, 2010) 
hanno trovato una forte correlazione tra le caratteristiche formali e funzionali dello scambio clinico, così come misurate dal DFA, e il contenuto delle narrazioni, così come interpretato alla luce dell'IMCS.

\section{Prospettive}

Nelle pagine precedenti abbiamo illustrato le principali linee di ricerca sul processo psicoterapeutico che ci hanno visto impegnati nell'ultimo lustro. Come è ovvio che sia, il nostro è un percorso in fieri. Ogni passo in avanti è foriero di ulteriori sollecitazioni e aperture: tre sono le principali aree sulle quali stiamo attualmente concentrando la nostra attenzione.

Sul piano teorico, alcuni di noi sono impegnati nell'approfondimento della linea di pensiero che ruota intorno alla generalizzazione abduttiva, nella prospettiva di fondare su tale modello di costruzione della conoscenza una rivisitazione in chiave idiografica della psicologia, dunque della psicologia clinica e della ricerca in psicoterapia (Salvatore, Gennaro, \& Valsiner, 2011).

Sul piano metodologico, riteniamo strategico il radicamento entro il campo della ricerca di processo della logica, dei modelli e degli strumenti di analisi derivanti dalla Teoria dei Sistemi Dinamici. Non si tratta di importare procedure di analisi e tecnicalità mutuate da altri campi, ma di sviluppare modelli dinamici coerenti con la natura dei fenomeni clinici (Salvatore \& Tschacher, submitted; Gelo, Ramseyer, Mergenthaler, \& Tschacher, 2008).

Infine, una parte rilevante dei nostri sforzi è indirizzata all'ulteriore sviluppo e validazione delle strategie di ricerca su cui abbiamo lavorato negli ultimi anni. Richiamiamo di seguito brevemente i programmi di lavoro su cui stiamo investendo.

1. Il primo è rappresentato dal DFA. Stiamo progettando una serie di ulteriori studi di casi, in ragione dei seguenti scopi: 
a) testare ulteriormente la validità di costrutto e convergente del metodo, in relazione ad altri metodi di analisi e nel contesto di una varietà di psicoterapie, terapeuti, pazienti, setting, esiti, caratteristiche del processo;

b) approfondire il significato clinico del metodo, analizzando il contenuto clinico associato agli andamenti degli indici dinamici e strutturali definiti dal metodo;

c) usare il DFA per differenziare gli aspetti della costruzione intersoggettiva del significato che operano come dimensioni costitutive dello scambio clinico rispetto agli aspetti che si caratterizzano come specifici - gli aspetti, cioè, che riflettono le caratteristiche associate alla efficacia ed efficienza del processo, e/o ad un tipo o altro di psicoterapia, così come agli aspetti contingenti e particolari dei diversi casi.

2. Il secondo programma di lavoro, avviato di recente, è l'elaborazione di un sistema di codifica degli interventi interpretativi del terapeuta: il GMI - Grid of Models of Interpretation (Auletta, 2010; Auletta \& Salvatore, 2008; Auletta, Salvatore, Metrangolo, Monteforte, Pace, \& Puglisi, submitted). Il nostro scopo è di pervenire a uno strumento di analisi focalizzato sulla funzione di regolazione dei processi di significazione esercitata dal terapeuta. Per questa ragione, il GMI è stato pensato come uno strumento trasversale ai diversi modelli di psicoterapia, complementare al DFA: attraverso di esso contiamo di rilevare il ruolo dell'attività regolativa (di negoziazione e di elaborazione dei significati) che il terapeuta esercita sulla dinamica dello scambio clinico.

Dal punto di vista metodologico, il GMI adotta un impianto coerente con l'approccio generale discusso nei precedenti paragrafi. La codifica si articola su 5 dimensioni (Contenuto dell'attività interpretativa, Dominio della attività interpretativa, Orientamento temporale, Orientamento spaziale, Forma), ciascuna organizzata in 


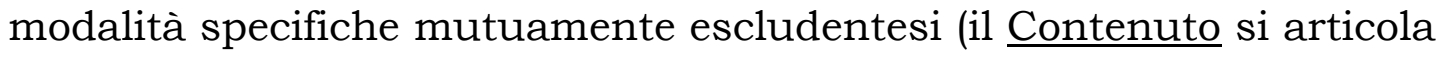
nelle modalità: Rappresentazione, Difese, Impulsi, Meccanismo di funzionamento, Affetti, Motivazione; il Dominio si articola nelle modalità: Intrapsichico - Relazionale; Orientamento temporale in: Presente, Passato, dal Presente al Passato, dal Passato al Presente; Orientamento Spaziale in: Interno ed Esterno al setting; Forma in: Assertiva, Soggettiva, Dimostrativa). Il GMI, dunque, non prende in esame l'intervento interpretativo del terapeuta in modo globale. Al contrario, esso opera analiticamente, focalizzandosi su 5 aspetti paralleli, ognuno dei quali preso in considerazione in quanto riconosciuto come una specifica dimensione/caratteristica dell'interpretazione. Successivamente, tramite una procedura di analisi multidimensionale, il GMI estrapola i pattern nei termini dei quali le diverse modalità si combinano nella concreta attività interpretativa sottoposta a investigazione.

Tale procedura presenta due vantaggi. In primo luogo, potenzia l'attendibilità della codifica, come conseguenza della specificità e del basso livello di inferenza implicato nella applicazione di ciascuna dimensione. In secondo luogo, questo tipo di approccio, grazie alla sua logica bottom-up permette di evitare il ricorso a griglie predefinite, pensate indipendentemente dal caso analizzato. Grazie a queste caratteristiche metodologiche, il GMI sembra essere in grado di offrire una descrizione del processo psicoterapeutico (specificamente, del processo interpretativo) sensibile al contesto dello scambio clinico (Greenberg \& Pinsof, 1986; Jones, Parke, \& Pulos, 1992; Lauro-Grotto, Salvatore, Gennaro, \& Gelo, 2009; PascualLeone, Greenberg \& Pascual-Leone, 2009).

Un primo studio ha applicato il GMI a 4 psicoterapie, 2 a indirizzo cognitivo e 2 a indirizzo psicodinamico (Auletta, 2010; Auletta, Salvatore, Metrangolo, Monteforte, Pace, \& Puglisi, submitted). I risultati ottenuti depongono in favore dell'attendibilità e della validità 
di costrutto del metodo. Il GMI ha messo in luce un livello soddisfacente di accordo tra giudici esperti, non influenzato dall'orientamento della terapia. Inoltre, le dimensioni e le categorie che costituiscono il sistema di codifica si sono dimostrate in grado di descrivere in maniera efficace le caratteristiche dell'attività interpretativa del terapeuta. Infine, sia a livello di singole categorie sia a livello di pattern aggregati, il GMI si è mostrato in grado di discriminare le psicoterapie analizzate in base al loro orientamento teorico e secondo criteri significativi da un punto di vista clinico.

3. Il terzo programma riguarda lo sviluppo di un metodo di analisi semantica delle narrazioni del paziente - il DMSC (Dynamic Mapping of the Structures of Content in Clinical Settings (Salvatore, Gennaro, Auletta, Grassi, \& Rocco, submitted). Tale metodo si focalizza su un livello generalizzato di significato concernente gli aspetti di base che organizzano le narrative (ad esempio, narrative relative al sé vs narrative relative ad altro da sé). Questa scelta è stata dettata da due fondamentali ragioni: da un lato, per ridurre la dipendenza dell'analisi dagli aspetti contingenti della comunicazione; dall'altro, anche in questo caso per fare del DMSC uno strumento trasversale ai diversi modelli di psicoterapia.

Il DMSC viene applicato da giudici ai trascritti delle sedute di psicoterapia e, secondo la stessa strategia metodologica del GMI (analisi dei pattern), è finalizzato a identificare le combinazioni (definite Pattern di contenuto) delle categorie che caratterizzano le narrative del paziente. Inoltre, coerentemente con le indicazioni metodologiche derivanti dalla Teoria dei Sistemi Dinamici, il DMSC non considera i pattern in sé, ma le loro transizioni: la probabilità che un determinato Pattern di contenuto succeda a un altro Pattern di contenuto.

Una prima ricerca (Salvatore, Gennaro, Auletta, Grassi, \& Rocco, submitted) ha applicato il DMSC ad un campione di 13 sedute 
estratte casualmente dalle 124 sedute del caso di Katja. I risultati di tale studio offrono sostegno alla validità di costrutto del metodo. In linea con il modello teorico su cui esso si basa, è stato riscontrato come: a) il DMSC delinea una rappresentazione significativa delle narrative del paziente in termini di Pattern di contenuto; b) alcune probabilità di transizione tra i pattern di contenuto (vale a dire la probabilità che ad un determinato pattern segua un certo altro pattern) sono associate in modo significativo alla qualità clinica delle sedute (definita indipendentemente sulla base di un criterio esterno).

Attraverso il DMSC intendiamo analizzare il contributo del paziente alla costruzione del dialogo terapeutico. Nelle nostre intenzioni il DMSC integra il repertorio degli strumenti grazie ai quali sviluppare un'analisi del processo terapeutico coerente con il quadro teorico definito dal TSSM. Riteniamo che l'applicazione congiunta del DFA, del GMI e del DMSC ci metterà nelle condizioni di analizzare il rapporto di reciproca regolazione tra la dinamica intersoggettiva di costruzione di senso e le operazioni discorsive prodotte dai partecipanti a tale dinamica (paziente e terapeuta).

\section{Bibliografia}

Andersen, S. (2001). The Emergence of Meaning: Generating Symbols from Random Sounds - A Factor Analytic Model. Journal of Quantitative Linguistics, 8(2), 101-136.

Auletta, A.F. (2010). The empirical study of therapist interpretations; the Grid of the Models of Interpretations (GMI). Tesi di dottorato non pubblicata, Università del Salento, Lecce.

Auletta, A.F., \& Salvatore, S. (2008). Grid of the Models of Interpretations. Manuale per la codifica. Manoscritto non pubblicato, Università del Salento, Lecce.

Auletta, A.F., Salvatore, S., Metrangolo, R., Monteforte, G., Pace, V., \& Puglisi, M. (submitted). The Grid of the Models of Interpretations (GMI): A trans-theoretical method to study therapist's interpretive activity.

Austin, J. (1962). How to do things with words. Oxford, UK: Oxford University Press. 
Ricerca in Psicoterapia / Research in Psychotherapy 2010; 2(13): 241-286 http://www.researchinpsychotherapy.net

Barkham, M., Stiles, W.B., \& Shapiro, D.A. (1993). The shape of change in psychotherapy: Longitudinal assessment of personal problems. Journal of Consulting and Clinical Psychology, 61, 667-677.

Bickhard, M.H. (2009). Interactivism: A Manifesto. New Ideas in Psychology, 27, 85-89.

Bruner, J. (1990). Acts of meaning. Cambridge: Harvard University Press.

Bucci, W. (1997). Psychoanalysis and Cognitive Science. New York: The Guildford Press.

Bühler, K. (1990). Theory of language. The representational function of language. Amsterdam/Philadelphia: John Benjamins Publishing Company (Versione originale pubblicata nel 1934).

Carli, R., \& Paniccia, R.M. (2002). L'analisi emozionale del testo. Uno strumento psicologico per leggere testi e discorsi. Milano: Franco Angeli.

Cole, M. (1996). Cultural Psychology. A once and future discipline. Cambridge: Harvard University Press.

Colli, A., \& Lingiardi, V. (2002). Indice di Valutazione dell'Alleanza Terapeutica. Una proposta di valutazione dell'alleanza terapeutica a partire dai trascritti delle sedute. In V. Lingiardi, (Ed.), L'Alleanza terapeutica. Teoria, clinica e ricerca (pp. 211-222). Milano: Raffaello Cortina.

Colli, A., \& Lingiardi, V. (2009). The Collaborative Interactions Scale: A new transcript-based method for the assessment of therapeutic alliance ruptures and resolutions in psychotherapy. Psychotherapy Research, 19(6), 718-734.

Dazzi, N. (2006). Il dibattito contemporaneo sulla ricerca in psicoterapia. In N. Dazzi, V. Lingiardi \& A. Colli (Eds.), La ricerca in psicoterapia. Modelli e strumenti (pp. 6-37). Milano: Raffaello Cortina.

Dazzi, N., Lingiardi, V., \& Colli, A. (Eds.). (2006). La ricerca in psicoterapia. Modelli e strumenti. Milano: Raffaello Cortina.

Di Nuovo, S. (2010). Elementare Watson. Criteri e metodi per l'investigazione. Enna: Città Nuova.

Dimaggio, G. (2007). Il terapeuta scannerizzato: commento alle analisi sul caso di Katja dal punto di vista del clinico. In G. Nicolò \& S. Salvatore (Eds.), La ricerca sui risultati e sul processo in psicoterapia (pp. 437-444). Roma: Firera \& Liuzzo Publishing Group.

Dimaggio, G., \& Semerari, A. (2001). El narcisismo en acción. Un modelo de psicopatologia ilustrado per el anilisis intensivo de un caso individual. Revista de Psicoterapia, 45.

Edwards, D., \& Potter, J. (1992). Discursive Psychology. London: Sage.

Forges Davanzati, G., Potì, S., \& Salvatore, S. (2008). L'economia irregolare come fenomeno culturale. Note per una interpretazione psicosociale. $\Pi$ pensiero economico moderno, XXVIII(4), 63-88.

Fornari, F. (1979). I fondamenti di una teoria psicoanalitica del linguaggio. Torino: Boringhieri.

Freud, S. (1900/1953). The Interpretation of Dreams. Harmondsworth: Penguin.

Gelo, O., \& Salvatore, S. (submitted). The dynamic nature of change in psychotherapy: Problematic assumptions and contrasting views.

Gelo, O., Ramseyer, F., Mergenthaler, E., \& Tschacher, W. (2008). Verbal coordination between patient and therapist speech: Hints for psychotherapy 
Ricerca in Psicoterapia / Research in Psychotherapy 2010; 2(13): 241-286 http://www.researchinpsychotherapy.net

process research. In: Society for Psychotherapy Research: Book of Abstracts of the 39th Annual Meeting, ULM: Ulmer Textbank, (pp. 90-91).

Gennaro, A. (2008). Improving "construct validity" in psychotherapy process research field: a research perspective. Tesi di dottorato non pubblicata, Università del Salento, Lecce.

Gennaro, A., Al-Radaideh, A., Gelo, O., Manzo, S., Nitti, M., \& Salvatore, S. (2010). Modelling psychotherapy process as sense-making dynamic: The Two Stage Semiotic Model (TSSM) and the Discourse Flow Analyzer (DFA). In S. Salvatore, J. Valsiner, J. Simon Travers \& A. Gennaro (Eds.), YIS: Yearbook of Idiographic Science (Volume 2) (pp. 131-169). Rome: Firera Publishing.

Gennaro, A., Goncalves, M.M., Mendes, I., Ribeiro, A., \& Salvatore, S. (in press). Dynamics of sense-making and development of the narrative in the clinical exchange. Research in Psychotherapy.

Gennaro, A., Melgiovanni, S., \& Serio, V. (2007, Giugno). Discorse Flow Analyzer: studio esplorativo di uno strumento per l'analisi discorsiva. Relazione presentata alla II Giornata Nazionale dei Giovani Ricercatori di Psicologia, Padova.

Gennaro, A., \& Salvatore, S. (in press). L'analisi automatizzata del contenuto. Uno strumento per gli interventi psicologici di prevenzione. In G. Savarese \& M. Cesaro (Eds.), Maltrattamenti ed abusi sessuali sui minori: fenomeno, tutela ed intervento. Lecce: Pensa Editore.

Gennaro, A., \& Salvatore, S. (submitted). Psychotherapy as dialog.

Gergen, K.J. (1999). An invitation to social construction. London: Sage.

Gonçalves, M.M., Matos, M., \& Santos, A. (2009). Narrative therapy and the nature of "innovative moments" in the construction of change. Journal of Constructivist Psychology, 22, 1-23.

Gonçalves, M.M., Ribeiro, A., Matos, M., Santos, A. \& Mendes (2010). The Innovative Moments Coding System: A methodological procedure for tracking changes in psychotherapy. In S. Salvatore, J. Valsiner, J. Simon Travers \& A. Gennaro (Eds.), YIS: Yearbook of Idiographic Science (Volume 2) (pp. 107-130). Rome: Firera Publishing.

Grassi, R. (2008). L'Analisi Frastica Multidimensionale. Ricerca sulla validità di costrutto. Tesi di dottorato non pubblicata, Università del Salento, Lecce.

Grasso, M. (2010). La relazione terapeutica. Bologna: Il Mulino.

Grasso, M., Salvatore S., \& Guido A. (2004). Una metodologia di analisi del processo psicoterapeutico: RIFLUD (rilevatore dei flussi discorsivi). Ricerca in Psicoterapia, 7(1), 35-50.

Greenberg, L.S. (1991). Research on the process of change. Psychotherapy Research, 1, 3-16.

Greenberg, L.S. (1994). The investigation of change: Its measurement and explanation. In R.L. Russel (Ed.), Reassessing psychotherapy research (pp. 114-143). New York/London: Guilford Press.

Greenberg, L.S., \& Pinsof, W.M. (Eds.). (1986). The psychotherapeutic process: A research handbook. New York: Guilford Press.

Harrè, R., \& Gillett, G. (1994). The discursive mind. London: Sage.

Jones, E.E., Parke, L.A., \& Pulos, S.M. (1992). How therapy is conducted in the private consulting room: A multidimensional description of brief psychodynamic treatments. Psychotherapy Research, 2, 16-30. 
Ricerca in Psicoterapia / Research in Psychotherapy 2010; 2(13): 241-286 http://www.researchinpsychotherapy.net

Lambert M.J. (2004). Bergin and Garfield's Handbook of Psychotherapy and Behavior Change (5th ed.). New York: Wiley and Sons.

Lamiell, J.T. (2003). Beyond individual and group differences. Thousand Oaks: Sage.

Lancia, F. (2002). The logic of a textscope. Retrivied 2007 August 18, from http://www.tlab.it/en/bibliography.php.

Laurenceau, J.P, Hayes, A.M., \& Feldman, G.C. (2007). Statistical and methodological issues in the study of change in psychotherapy. Clinical Psychology Review, 27, 682-695.

Lauro-Grotto, R., Salvatore, S., Gennaro, A., \& Gelo, O. (2009). The unbearble dynamicity of psychological processes: highlights of the psychodynamics theories. In J. Valsiner, P. Molenaar, M. Lyra \& N. Chaudhary (Eds.), Dynamics process methodology in the social and developmental sciences (pp. 1-30). New York: Springer.

Lingiardi, V., Colli, A., \& Gazzillo, F. (2007). Diagnosi di personalità, alleanza terapeutica e meccanismi di difesa. In G. Nicolò \& S. Salvatore (Eds.), La ricerca sui risultati e sul processo in psicoterapia (pp. 239-250). Roma: Edizioni Carlo Amore.

Lutz, W., \& Hill, C.E. (2009). Special section: Quantitative and qualitative methods for psychotherapy research. Psychotherapy Research, 19, 369611.

Manzo, S. (2010). La ricerca di processo nel decennio 1998-2007. Ricerca in psicoterapia/Research in psychotherapy: Psychopathology, Process and Outcome, 13(1), 92-119. Available from http:/ / www.researchinpsychotherapy.net.

Matos, M., Santos, A., Gonçalves, M., \& Martins, C. (2009). Innovative moments and change in narrative therapy. Psychotherapy Research, 19(1), 68-80.

Mergenthaler, E. (1996). Emotion abstraction patterns in verbatim protocols: A new way of describing therapeutic processes. Journal of Consulting and Clinical Psychology, 64, 1306-1318.

Molenaar, P.C.M. (2004). A manifesto on psychology as idiographic science: Bringing the person back into scientific psychology, this time forever. Measurement, 2(4), 201-218.

Molenaar, P.C.M., \& Valsiner, J. (2009). How generalization works through the single case: a simple idiographic process analysis of an individual psychotherapy. In S. Salvatore, J. Valsiner, S. Strout \& J. Clegg (Eds.), YIS: Yearbook of Idiographic Science (Volume 1) (pp. 23-38). Rome: Firera Publishing Group.

Nitti, M., Ciavolino, E., Salvatore, S., \& Gennaro, A. (2010). Analyzing psychotherapy process as intersubjective sensemaking. An approach based on discourse analysis and neural networks. Psychotherapy Research, 2O(5), 546-563.

Pascual-Leone, A., Greenberg, L.S., \& Pascual-Leone, L. (2009). Developments in task analysis: New methods to study change. Psychotherapy research, 19(4), 527-542.

Perry, J.C. (1990). Defense Mechanism Rating Scale. Unpublished manuscript, Harvard Medical School of Boston.

Russell, B. (1994). Meinong's theory of complexes and assumptions. Mind, 13, 204-219. 
Ricerca in Psicoterapia / Research in Psychotherapy 2010; 2(13): 241-286 http://www.researchinpsychotherapy.net

Salvatore, S. (2006a). Modelli di conoscenza e agire psicologico/Models of knowledge and psychological action. Rivista di Psicologia Clinica, 2-3. Retrieved from http://www.rivistadipsicologiaclinica. it/english/number2/Salvatore.htm.

Salvatore, S., (2006b). Intorno ad alcune premesse della ricerca empirica in psicoterapia. Ricerca in psicoterapia, 9(2), 261-280.

Salvatore, S. (in press). Social life of the sign: Sensemaking in society. In J. Valsiner (Ed.), The Oxford Handbook of Culture and Psychology. Oxford: Oxfor Press.

Salvatore, S., \& Freda, M.F. (2010). The role of the unconscious in communication. Outlines for a comprehensive model of sensemaking. New Ideas in Psychology. Available from doi: 10.1016/j.newideapsych.2010.06.001.

Salvatore, S., Freda, M.F., Ligorio, B., Iannaccone, A., Rubino, F., Scotto di Carlo, M., Bastianoni, P., \& Gentile, M. (2003). socioconstructivism and theory of the unconsious. A gaze over a research horizon. European Journal of School Psychology, 1(1), 9-36.

Salvatore, S., Forges Davanzati, G., Potì, S., \& Ruggeri, R. (2009). Mainstream Economics and sense-making. Integrative Psychological and Behavioral Science, 43(2), 158-177.

Salvatore, S., Gelo, O., Gennaro, A., \& Manzo, S. (2009). Looking at the psychotherapy process as an intersubjective dynamic of sensemaking. A case study with Discourse Flow Analysis. Journal of Constructivist Psychology, 23(3), 195-230.

Salvatore, S., Gennaro, A., Auletta, A., Grassi, R., \& Rocco, D. (submitted). Dynamic Mapping of the Structures of Content in Clinical Settings (DMSC). A new coding system for analyzing the patient's narratives.

Salvatore, S., Gennaro, A., Auletta, A., Tonti, M., \& Nitti, M. (submitted). Automated method of content analysis. A device for psychotherapy process research.

Salvatore, S., Gennaro, A., Grassi, R., Manzo, S., Melgiovanni, S., Mossi, P., Olive, C., \& Serio, V. (2007). La dinamica discorsiva dello scambio terapeutico come indice clinico. Applicazione del DFA (Discursive Flow Analyzer) alla psicoterapia di Katja. In G. Nicolò \& S. Salvatore (Eds.), La ricerca sui risultati e sul processo in psicoterapia (pp. 335-397). Roma: Edizioni Carlo Amore.

Salvatore, S., Gennaro, Lis, A., Di Riso, D., Laghezza, L., \& Sbabo, S. (2006). Process research: Defence mechanisms trend in a psychodynamically oriented supportive psychotherapy. In: Society for Psychotherapy Research, Book of Abstracts 37th Annual Meeting (p. 162). Ulm: Ulmer Textbank.

Salvatore, S., Gennaro, A., Manzo, S., Melgiovanni, S., \& Serio, S. (2007, March). The DFA (Discourse Flow Analyzer) A study of its convergent validity. Paper presented at the $7^{\text {th }}$ European Conference on Psychotherapy Research, Madeira, Portugal.

Salvatore, S., Gennaro, A., \& Valsiner, J. (2011). YIS: Yearbook of idiographic science (Volume 4). Charlotte, NC: Information Age Publishing.

Salvatore, S., Grasso, M., \& Tancredi, F. (2004, March). A methodology of process analysis: Discourse Flows Reader (CFR). A pilot study. Paper presented at the $2^{\text {nd }}$ Joint Meeting of the SPR European and UK Chapters, Lausanne, Switzerland. 
Ricerca in Psicoterapia / Research in Psychotherapy 2010; 2(13): 241-286 http://www.researchinpsychotherapy.net

Salvatore, S., Lauro-Grotto, R., Gennaro, A., \& Gelo, O. (2008). Per una psicologia dinamica dinamica. In S. di Nuovo e G. Falgares (Eds.), Per una psicologia psicologica. Scritti in onore di Franco Di Maria (pp. 377-397). Milano: Franco Angeli.

Salvatore, S., Lauro-Grotto, R., Gennaro, A., \& Gelo, O. (2009). Attempts to grasp the dynamicity of intersubjectivity. In J. Valsiner, P.C.M. Molenaar, M.C.P. Lyra \& N. Chaudhary (Eds.), Dynamic process methodology in the social and developmental sciences (pp. 171-190). New York: Springer.

Salvatore, S., Ligorio, M.B. \& De Franchis, C. (2005). Does Psychoanalytic Theory Have Anything to Say on Learning? European Journal of School Psychology, 3(1), 101-126.

Salvatore, S., Mossi, P., \& Gennaro, A. (2007). Il discorso sulla psicoterapia, tra dinamiche istituzionali, concezioni della mente, modelli di intervento, pratiche di ricerca. In G. Nicolò \& S. Salvatore (Eds.), La ricerca sui risultati e sul processo in psicoterapia. Roma: Edizioni Carlo Amore.

Salvatore, S., Serio, V., \& Manzo S. (2006, Settembre). Studio del processo terapeutico di una Psicoterapia Cognitiva mediante DFA. Relazione presentata al Convegno Nazionale della Società per la Ricerca in Psicoterapia, Reggio Calabria.

Salvatore, S., Tebaldi, C., \& Poti, S. (2006/2009). The discursive dynamic of sensemaking. In S. Salvatore, J. Valsiner, S. Strout, \& J. Clegg (Eds.), YIS: Yearbook of idiographic science (Vol. 1) (pp. 39-72). Rome: Firera Publishing.

Salvatore, S., \& Tschacher, W. (submitted). Time dependency of psychotherapeutic exchanges: The contribution of the Theory of Dynamic Systems in analyzing process.

Salvatore, S., \& Valsiner, J. (2006). 'Am I really a psychologist?' Making sense of a super-human social role. European Journal of School Psychology, 4, 5-30.

Salvatore, S., \& Valsiner, J. (2009). Idiographic Science on its Way: Towards Making Sense of Psychology", In S. Salvatore, J. Valsiner, S. Strout \& J. Clegg (Eds.), Yearbook of Idiographic Science (Volume 1) (pp. 9-19). Roma: Firera Publishing Group.

Salvatore, S., \& Valsiner, J. (2010). Idiographic science as a non-existing object: the importance of the reality of the dynamic system. In S. Salvatore, J. Valsiner, J. Simon Travers \& A. Gennaro (Eds.), YIS: Yearbook of Idiographic Science (Volume 3) (pp. 7-27). Roma: Firera Publishing.

Salvatore, S., \& Valsiner, J. (in press). Between the General and the Unique: Overcoming the nomothetic versus idiographic opposition, Theory and Psychology.

Salvatore, S., Valsiner, J., Strout, S., \& Clegg, J. (2009). YIS: Yearbook of idiographic science (Volume 1). Roma: Firera Publishing Group.

Salvatore, S., Valsiner, J., Travers-Simon, J., \& Gennaro, A. (2010a). YIS: Yearbook of idiographic science: (Volume 2). Roma: Firera Publishing Group.

Salvatore, S., Valsiner, J., Travers-Simon, J., \& Gennaro A. (2010b). YIS: Yearbook of idiographic science: (Volume 3). Roma: Firera Publishing Group. 
Ricerca in Psicoterapia / Research in Psychotherapy 2010; 2(13): 241-286 http://www.researchinpsychotherapy.net

Salvatore, S., \& Venuleo, C. (2008). Understanding the role of emotion in sensemaking. A semiotic psychoanalytic oriented perspective. Integrative Journal of Psychological and Behavioral Science, 42, 32-46.

Salvatore, S., \& Venuleo, C. (2010). The unconscious as source of sense: A psychodynamic approach to meaning. In B. Wagoner (Ed.), Symbolic Transformations: The mind in movement through culture and society (pp. 59-74). London: Routledge.

Salvatore, S., \& Zittoun, T. (in press). Outlines of a psychoanalytically informed cultural psychology. In S. Salvatore, T. Zittoun (Eds.), Cultural Psychology and Psychoanalysis in Dialogue. Issues for Constructive Theoretical and Methodological Synergies. Charlotte, NC: Information Age Publishing.

Santos, A., Goncalves, M.M., Matos, M., \& Salvatore, S. (2009). Innovative moments and change pathways. A good outcome case of narrative therapy. Psychology and Psychotherapy: Theory, Research and Practice, 82, 449466.

Smedslund, J. (1987). The epistemic status of inter-item correlations in Eysenck's Personality Questionnaire. Scandinavian Journal of Psychology, $28,42-55$.

Stiles, W.B., \& Shapiro, D.A. (1994), Disabuse of the drug metaphor: Psychotherapy process-outcome correlations. Journal of Consulting and Clinical Psychology, 62(5), 942-948.

Toomela, A. (2009). How methodology became a toolbox-and how it escapes from that box. In J. Valsiner, P. Molenaar, M. Lyra \& N. Chaudhary (Eds.), Dynamic process methodology in the social and developmental sciences (pp. 45-66). New York: Springer.

Toomela, A. (2010). The methodology of idiographic science: The limits of single-case studies and the role of typology. In S. Salvatore, J. Valsiner, J. Simon Travers \& A. Gennaro (Eds), YIS: Yearbook of Idiographic Science (Volume 2) (pp. 13-33). Rome: Firera Publishing.

Valsiner, J. (2007). Culture in Minds and Societies. Foundations of Cultural Psychology. New Delhi: Sage.

Valsiner, J., \& Rosa, A. (2007). The Cambridge Handbook of Sociocultural Psychology. Cambridge: Cambridge University Press.

Venuleo, C., \& Salvatore, S. (2008). Una lettura semiotica del gioco d'azzardo. In G. Lavanco \& M. Croce (Eds.), Psicologia delle dipendenze sociali (pp. 299-333). Milano: McGraw-Hill.

Von Eye, A., Mum, E.Y., \& Mair, P. (2009). What carries a mediation process. Configurational analysis of mediation. Integrative Psychological and Behavioral Science, 43(3), 228-247.

Windelband, W. (1998). History and natural science. Theory \& Psychology, 8, 5-22. (Versione originale pubblicata nel 1904).

Wittgenstein, L. (1958). Philosophical investigations. Oxford, UK: Basic Blackwell. 


\section{Abstract}

The paper presents the three main lines of work on the process research the Authors' interest is focused on: a) the conceptual analysis of the theoretical and methodological assumptions grounding the research in the field; b) the definition of a general model of the clinical process; c) the development of strategies of analysis of the clinical exchange consistent with that general model. The conceptual framework, the main results and the future directions of each line are discussed.

\section{Keywords}

Process research, theory of dynamic systems, idiographic science, abduction, textual analysis, Discursive Flow Analys 